\title{
Asymptotic size of Herman rings of the complex standard family by quantitative quasiconformal surgery
}

\author{
NÚRIA FAGELLA $\dagger$, TERE M. SEARA $\ddagger$ and JORDI VILLANUEVA $\ddagger$ \\ $\dagger$ Departament de Matemàtica Aplicada i Anàlisi, Universitat de Barcelona, \\ Gran Via 585, 08007 Barcelona, Spain \\ (e-mail:fagella@maia.ub.es) \\ \$Departament de Matemàtica Aplicada I, Universitat Politècnica de Catalunya, \\ Diagonal 647, 08028 Barcelona, Spain \\ (e-mail:tere.m-seara@upc.es, jordi@vilma.upc.es)
}

(Received 23 May 2003 and accepted in revised form 13 January 2004)

\begin{abstract}
In this paper we consider the complexification of the Arnold standard family of circle maps given by $\widetilde{F}_{\alpha, \varepsilon}(u)=u e^{i \alpha} e^{(\varepsilon / 2)(u-1 / u)}$, with $\alpha=\alpha(\varepsilon)$ chosen so that $\widetilde{F}_{\alpha(\varepsilon), \varepsilon}$ restricted to the unit circle has a prefixed rotation number $\theta$ belonging to the set of Brjuno numbers. In this case, it is known that $\widetilde{F}_{\alpha(\varepsilon), \varepsilon}$ is analytically linearizable if $\varepsilon$ is small enough and so it has a Herman ring $\widetilde{U}_{\varepsilon}$ around the unit circle. Using Yoccoz's estimates, one has that the size $\widetilde{R}_{\varepsilon}$ of $\widetilde{U}_{\varepsilon}$ (so that $\widetilde{U}_{\varepsilon}$ is conformally equivalent to $\left\{u \in \mathbb{C}: 1 / \widetilde{R}_{\varepsilon}<\right.$ $\left.|u|<\widetilde{R}_{\varepsilon}\right\}$ ) goes to infinity as $\varepsilon \rightarrow 0$, but one may ask for its asymptotic behavior.

We prove that $\widetilde{R}_{\varepsilon}=(2 / \varepsilon)\left(R_{0}+\mathcal{O}(\varepsilon \log \varepsilon)\right)$, where $R_{0}$ is the conformal radius of the Siegel disk of the complex semistandard map $G(z)=z e^{i \omega} e^{z}$, where $\omega=2 \pi \theta$. In the proof we use a very explicit quasiconformal surgery construction to relate $\widetilde{F}_{\alpha(\varepsilon), \varepsilon}$ and $G$, and hyperbolic geometry to obtain the quantitative result.
\end{abstract}

\section{Introduction}

The complex standard family of self maps of $\mathbb{C}^{*}=\mathbb{C} \backslash\{0\}$ is given by the two-parameter family

$$
\widetilde{F}_{\alpha, \varepsilon}(u)=u e^{i \alpha} e^{(\varepsilon / 2)(u-1 / u)},
$$

where $\alpha \in[0,2 \pi)$ and $\varepsilon \in[0,1)$. These maps are holomorphic in $\mathbb{C}^{*}$ and the points at 0 and infinity are essential singularities (see [Ba, Ko1, Mak, Ke, Ko2, F]). For small $\varepsilon$, these functions are perturbations of the rotation of angle $\alpha$ with respect to the origin. The interest in this family relies on the fact that it is the extension to the complex plane of the well-known Arnold family of circle maps (see [Ar, $\mathbf{d M v S}]$ ). Indeed, the unit circle $\mathbf{C}_{1}$ 


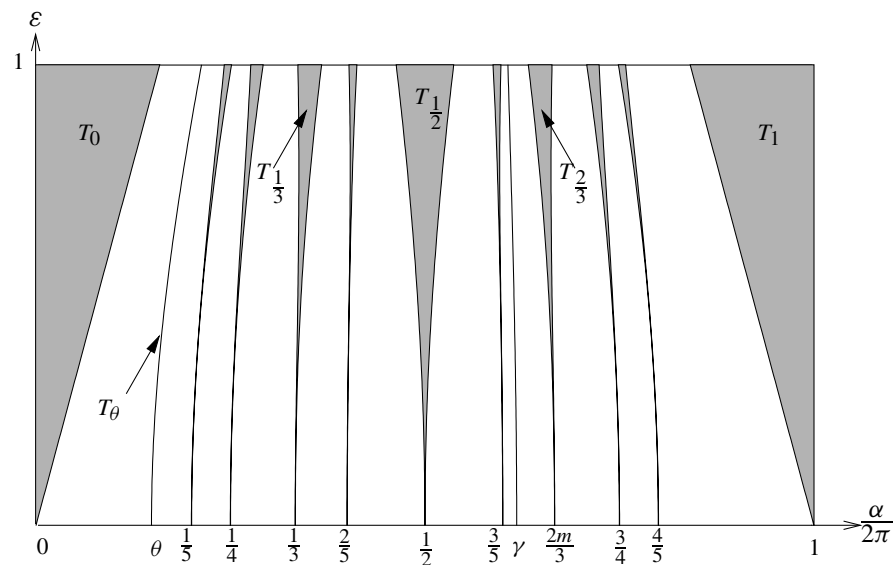

FIGURE 1. Rational Arnold tongues in the parameter space of the standard family up to denominator 5 (note that these are sets with interior). The curves correspond to the irrational tongues for $\gamma=(\sqrt{5}-1) / 2$ and $\theta=\sqrt[5]{2}-1$.

is invariant under $\widetilde{F}_{\alpha, \varepsilon}$, and using the homeomorphism $e^{2 \pi i x}$ between $\mathbb{T}^{1}=\mathbb{R} / \mathbb{Z}$ and $\mathbf{C}_{1}$, the map $\widetilde{F}_{\alpha, \varepsilon \mid \mathbf{C}_{1}}$ becomes the Arnold family:

$$
\begin{aligned}
\tilde{f}_{\alpha, \varepsilon}: \mathbb{T}^{1} & \longrightarrow \mathbb{T}^{1} \\
x & \longrightarrow x+\frac{\alpha}{2 \pi}+\frac{\varepsilon}{2 \pi} \sin (2 \pi x) .
\end{aligned}
$$

It is clear that if $\varepsilon \in[0,1), \widetilde{f}_{\alpha, \varepsilon}$ is an orientation-preserving diffeomorphism of $\mathbb{T}^{1}$, and, thus, for each pair of parameters $(\alpha, \varepsilon)$ the rotation number of $\widetilde{f}_{\alpha, \varepsilon}$ is well defined (see $\$ 2.1$ for the definition of the rotation number). The rotation number measures the asymptotic rate of rotation of points of the circle. For instance, a rigid rotation of $\mathbb{T}^{1}$ of the form $\mathcal{T}_{\theta}(x)=x+\theta$ has rotation number $\theta$. All throughout this paper we fix an irrational rotation number $\theta$ and always choose the parameters $\alpha$ and $\varepsilon$ such that the rotation number of $\widetilde{f}_{\alpha, \varepsilon}$ is $\theta$. More precisely, we choose our rotation number $\theta$ among the Brjuno set of irrational numbers, which contains all Diophantine numbers (see [PM] for a precise definition of these sets). In the $(\alpha, \varepsilon)$-parameter space, the set of parameters with a given rotation number $\theta$ is called the Arnold tongue $T_{\theta}$. If $\theta$ is rational, its Arnold tongue is a set with interior, while if the rotation number is an irrational number $\theta$, then $T_{\theta}$ corresponds to a curve connecting $\varepsilon=0$ and $\varepsilon=1$, which is in fact the graph of a function $\varepsilon \mapsto \alpha(\varepsilon)$ with $\alpha(0)=2 \pi \theta$ (see Figure 1). If $\theta$ is a Brjuno number, the curve $\alpha(\varepsilon)$ is known to be analytic for $\varepsilon$ small enough [Ri, FG].

Moreover, if the rotation number $\theta$ is a Brjuno number we have that for $\varepsilon$ small enough, the map $\widetilde{f}_{\alpha(\varepsilon), \varepsilon}$ is analytically linearizable (see [Y2, PM, Ri]). That is, there is an analyc $\operatorname{map} \widetilde{\eta}_{\varepsilon}: \mathbb{T}^{1} \rightarrow \mathbb{T}^{1}$ that conjugates $\widetilde{f}_{\alpha(\varepsilon), \varepsilon}$ to $\mathcal{T}_{\theta}$, i.e.

$$
\tilde{f}_{\alpha(\varepsilon), \varepsilon} \circ \tilde{\eta}_{\varepsilon}=\tilde{\eta}_{\varepsilon} \circ \mathcal{T}_{\theta} .
$$

Equivalently, to say that $\widetilde{F}_{\alpha(\varepsilon), \varepsilon}$ restricted to $\mathbf{C}_{1}$ is analytically linearizable means that there is an analytic map $\widetilde{\varphi}_{\varepsilon}: \mathbf{C}_{1} \rightarrow \mathbf{C}_{1}$, such that

$$
\widetilde{F}_{\alpha(\varepsilon), \varepsilon} \circ \widetilde{\varphi}_{\varepsilon}=\widetilde{\varphi}_{\varepsilon} \circ \mathcal{R}_{\omega},
$$


where $\mathcal{R}_{\omega}(u)=e^{i \omega} u$ and $\omega=2 \pi \theta$. Since the linearization $\widetilde{\varphi}_{\varepsilon}$ is analytic, it can be extended to a neighborhood of the unit circle of the form $A(1 / r, r)$, where we define

$$
A\left(r_{1}, r_{2}\right)=\left\{u \in \mathbb{C}: r_{1}<|u|<r_{2}\right\}
$$

as the straight ring of radii $r_{1}$ and $r_{2}$. We denote by $\widetilde{A}_{\varepsilon}=A\left(1 / \widetilde{R}_{\varepsilon}, \widetilde{R}_{\varepsilon}\right)$ the maximal ring for which $\widetilde{\varphi}_{\varepsilon}$ can be analytically continued. Then, it is easy to check that being $\widetilde{F}_{\alpha(\varepsilon), \varepsilon \mid \mathbf{C}_{1}}$ analytically linearizable is equivalent to the existence of a Herman ring $\widetilde{U}_{\varepsilon}$ for $\widetilde{F}_{\alpha(\varepsilon), \varepsilon}$, which is given by $\widetilde{U}_{\varepsilon}:=\widetilde{\varphi}_{\varepsilon}\left(\widetilde{A}_{\varepsilon}\right)$. In $\widetilde{U}_{\varepsilon}$, every orbit under $\widetilde{F}_{\alpha(\varepsilon), \varepsilon}$ lies on an invariant closed curve which has rotation number $\theta$. Since $\widetilde{\varphi}_{\varepsilon}$ is unique (up to composition with rotations), the constant $\widetilde{R}_{\varepsilon}$ is univocally defined and we call it the size of the Herman ring.

The main goal of this paper is to give an asymptotic estimate for the size $\widetilde{R}_{\mathcal{\varepsilon}}$ of the Herman $\operatorname{ring} \widetilde{U}_{\varepsilon}$ as $\varepsilon \rightarrow 0$.

The sharpest results concerning the size of Herman rings for univalent maps on a given ring are due to Yoccoz (see Theorems 2.1 and 2.3), who gives an estimate that can be applied to any analytic map $F$ that leaves the unit circle $\mathbf{C}_{1}$ invariant and has rotation number $\theta$ (i.e. a lift on $\mathbb{R}$ of $F_{\mid \mathbf{C}_{1}}$ has rotation number $\theta$ ), and which depends only on $\theta$ and on the size of the domain where the map is univalent. In $\S 3$ we will see that this general result applied to the complex standard family leads to

$$
\widetilde{R}_{\varepsilon} \geq K \frac{\sigma(\varepsilon)}{\varepsilon},
$$

where $K=\exp \left(-\Phi(\theta)-2 \pi C_{0}\right)$, $\Phi$ is the Brjuno function [MMY], $C_{0}$ is a universal constant and $\sigma(\varepsilon)=1+\sqrt{1-\varepsilon^{2}}$ is defined in such a way that $\widetilde{F}_{\alpha(\varepsilon), \varepsilon}$ is univalent in $A(\varepsilon / \sigma(\varepsilon), \sigma(\varepsilon) / \varepsilon)$.

The fact that this estimate holds for any analytic diffeomorphism having $\mathbf{C}_{1}$ invariant with rotation number $\theta$ and univalent at least in $A(\varepsilon / \sigma(\varepsilon), \sigma(\varepsilon) / \varepsilon)$, suggests that a better estimate can be found for the complex standard family. We shall return to this problem in a moment, but first let us consider what is known as the complex semistandard map of parameter $e^{i \omega}$

$$
G(z)=z e^{i \omega} e^{z}
$$

Observe that $z=0$ is a fixed point of $G$ with derivative $e^{i \omega}$. Since $\omega=2 \pi \theta$, and $\theta$ is a Brjuno number, it is known [Br1, Br2] that $G$ has a Siegel disk around the origin, which we denote by $U$. This means that if we call $\mathbb{D}_{r}$ the open disk of center 0 and radius $r$, there exists a unique maximal number $R_{0}>0$ and a unique conformal isomorphism

$$
\varphi: \mathbb{D}_{R_{0}} \longrightarrow U, \quad \varphi(0)=0, \quad \varphi^{\prime}(0)=1
$$

that conjugates $G$ to the rotation $\mathcal{R}_{\omega}$, i.e. $G \circ \varphi=\varphi \circ \mathcal{R}_{\omega}$. The number $R_{0}$ is known as the conformal radius of the Siegel disk. Standard arguments show that $R_{0}$ is always finite. Lower bounds for $R_{0}$ (as a function of $\omega$ ) could be obtained applying Yoccoz's results [Y1] to the semistandard map.

We now return to the problem of estimating the size of the Herman ring $\widetilde{U}_{\varepsilon}$. Our main result is the following theorem.

THEOREM A. Let $\theta$ be a Brjuno number and consider the standard map $\widetilde{F}_{\alpha, \varepsilon}(u)=$ $u e^{i \alpha} e^{(\varepsilon / 2)(u-1 / u)}$, with $\alpha=\alpha(\varepsilon)$ such that $\widetilde{F}_{\alpha(\varepsilon), \varepsilon}$ restricted to $\mathbf{C}_{1}$ has rotation number $\theta$. 
Let $\widetilde{R}_{\varepsilon}$ be the size of its Herman ring and let $R_{0}$ be the conformal radius of the Siegel disk of the semistandard map $G(z)=z e^{i \omega} e^{z}$, where $\omega=2 \pi \theta$. Then,

$$
\widetilde{R}_{\varepsilon}=\frac{2}{\varepsilon}\left(R_{0}+\mathcal{O}(\varepsilon \log \varepsilon)\right)
$$

Remark 1.1. We believe that this is the best estimate that can be obtained with our methods. However, some recent developments (work in progress) seem to indicate that a more optimal estimate could be $\widetilde{R}_{\varepsilon}=(2 / \varepsilon)\left(R_{0}+\mathcal{O}\left(\varepsilon^{2}\right)\right.$ ) (with $\widetilde{R}_{\varepsilon}$ analytic on $\varepsilon$ ). As a vague indication, this could follow from knowing that the complexification of the Arnold tongue $T_{\theta}$, with $\theta$ a Brjuno number, can be parametrized holomorphically by a complex parameter strongly related with the modulus of the ring.

An analogue of Theorem A, for Chirikov's standard and semistandard maps of $\mathbb{R}^{2}$, was proved in [SV] using KAM methods and complex matching, and therefore restricting the result to Diophantine rotation numbers. In the present paper, we prove Theorem A using quasiconformal surgery, inspired by a qualitative construction of Geyer in [G] which relates the standard and the semistandard maps. We modify this construction by introducing the dependence on the parameter $\varepsilon$ and by making most of its ingredients completely explicit. These additions will give us the possibility of obtaining quantitative estimates from the geometric construction.

From all the partial results involved in the proof of Theorem A we choose the following to be remarked on here, because of its interest in itself and its possible use in other surgery constructions. Part (a) is known from a theorem of Teichmüller, and relates the hyperbolic distance between the Morrey-Bojarski-Ahlfors-Bers map (see Theorem 2.12) and the identity with the dilatation $\|\mu\|$. From this result, in part (b) we obtain an estimate for how close this map is from the identity.

Throughout the paper, $\delta_{\mathcal{U}}$ indicates the hyperbolic distance inside $\mathcal{U}$, where $\mathcal{U}$ is a hyperbolic set (see $\$ 2.3$ ). The notation $\|\cdot\|$ denotes the infinity norm and $\mathbb{D}=\mathbb{D}_{1}$.

Proposition B. Let $\mu$ be a Beltrami coefficient on $\mathbb{C}($ see $\$ 2.2)$ and $h: \mathbb{C} \rightarrow \mathbb{C}$ be the unique quasiconformal solution of the Beltrami equation $\partial \mathrm{h} / \partial \bar{z}=\mu(\partial \mathrm{h} / \partial z)$ fixing 0 and 1 (see Theorem 2.12).

(a) For any $z \in \mathbb{C} \backslash\{0,1\}$, we have $\delta_{\mathbb{C} \backslash\{0,1\}}(z, h(z)) \leq \delta_{\mathbb{D}}(0,\|\mu\|)$.

(b) There exists a universal constant $0<\rho<1$ such that if $\|\mu\| \leq \rho$, then for any $z \in \mathbb{D}_{\rho}^{*}=\mathbb{D}_{\rho} \backslash\{0\}$ verifying $\|\mu\||\log | z|| \leq \rho$, one has

$$
|h(z)-z| \leq C\|\mu\||z||\log | z||
$$

where $C>0$ only depends on $\rho$.

This paper is organized as follows. Section 2 contains basic introductions to some of the tools and preliminary results that will be used during the proofs of Theorem A and Proposition B. The expert reader can go directly to $\S 3$, where the problem is scaled and restated more precisely. Sections 4 and 5 contain the actual proofs of Theorem A and Proposition B, respectively. 


\section{Preliminaries}

In this section, we state the basic results that we need to prove Theorem $\mathrm{A}$ and Proposition B. In $\$ 2.1$ we review previous results about the linearization of analytic circle maps and their translation to maps of the complex plane having an invariant circle. Section 2.2 is devoted to quasiconformal mappings and measurable Riemann mapping theorem. Finally, in $\$ 2.3$ we give some definitions and results in hyperbolic geometry.

2.1. Analytic linearization. Let $f: \mathbb{T}^{1} \rightarrow \mathbb{T}^{1}$ be an orientation-preserving homeomorphism of the circle $\mathbb{T}^{1}=\mathbb{R} / \mathbb{Z}$, and $f_{0}$ its lift to $\mathbb{R}$ with the normalization $f_{0}(0) \in[0,1)$. To such a map one can assign a rotation number defined as

$$
\rho(f)=\lim _{n \rightarrow \infty} \frac{f_{0}^{n}(x)-x}{n}
$$

where $x$ is any point in $\mathbb{R}$. It is well known (see e.g. [dMvS]) that $f$ being a homeomorphism guarantees that this limit exists and is independent of the point $x$. With this definition, $\rho(f)$ is a rational number if and only if $f$ has a periodic orbit. We are interested in maps with an irrational rotation number.

If the rotation number of $f$ is an irrational number $\theta$ and $f \in \mathcal{C}^{2}\left(\mathbb{T}^{1}\right)$, Denjoy's theorem (see $[\mathbf{d M v S}]$ ) ensures that $f$ is topologically conjugate to the rigid rotation of angle $\theta$, $\mathcal{T}_{\theta}(x)=x+\theta$. That is, there exists a homeomorphism $\eta: \mathbb{T}^{1} \rightarrow \mathbb{T}^{1}$ such that $\eta \circ \mathcal{T}_{\theta}=f \circ \eta$, making the following diagram commute:

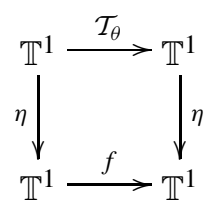

If we require $\eta(0)=0$, then the conjugacy is unique.

From now on we restrict ourselves to the case where $f$ is an analytic diffeomorphism of $\mathbb{T}^{1}$, and therefore it can be extended to a complex annulus, of certain width $\Delta>0$, around $\mathbb{T}^{1}$ :

$$
\mathcal{A}_{\Delta}=\{z \in \mathbb{C} / \mathbb{Z}:|\operatorname{Im}(z)|<\Delta\} .
$$

Abusing notation, we again denote this extension by $f$. If the conjugacy $\eta$ is also analytic, the map $f$ is said to be analytically linearizable. Then again, $\eta$ can be extended to a neighborhood of the circle, and it is easy to check (by the principle of analytic continuation) that its extension also conjugates $f$ to $\mathcal{T}_{\theta}$ wherever $\eta$ is defined.

We are particularly interested in the case where $F: \mathcal{U} \subset \mathbb{C} \rightarrow \mathbb{C}$ is an analytic map having the unit circle $\mathbf{C}_{1}$ invariant, and $f$ is the map on $\mathbb{T}^{1}$ induced by $F_{\mid \mathbf{C}_{1}}$. In this case, we say that $F_{\mid \mathbf{C}_{1}}$ is analytically linearizable if there exists an analytic diffeomorphism $\varphi: \mathbf{C}_{1} \rightarrow \mathbf{C}_{1}$, such that $\varphi \circ \mathcal{R}_{\omega}=F \circ \varphi$, where $\mathcal{R}_{\omega}(u)=e^{i \omega} u$ and $\omega=2 \pi \theta$. If we ask $\varphi(1)=1, \varphi$ is univocally defined and the relation between $\eta$ and $\varphi$ is given by

$$
\varphi\left(e^{2 \pi i x}\right)=e^{2 \pi i \eta(x)}, \quad x \in \mathbb{T}^{1} .
$$

The image by $\varphi$ of the maximal ring where $\varphi$ can be analytically continued is called the Herman ring of $F$. If $R$ is the outer radius of this ring (in the understanding that this ring is 
symmetric with respect to the unit circle, and then it is of the form $A(1 / R, R))$, the width of the annulus of analyticity of $f$ around $\mathbb{T}^{1}$ is $(1 / 2 \pi) \log R$. The quantity $(1 / \pi) \log R$ is called the modulus of the ring and we call $R$ the size of the ring.

Arnold showed in [Ar] that if $\theta$ is a Diophantine number and $f$ is close enough to the rigid rotation $\mathcal{T}_{\theta}$, then $f$ is analytically linearizable. This result was later improved by Rüssmann [Ru1, Ru2], Herman [Her1, Her2] and Yoccoz [Y2, PM]. The sharpest results are due to Yoccoz and we state them below. In the statement, $\Phi: \mathbb{R} \backslash \mathbb{Q} \rightarrow \mathbb{R}^{+} \cup\{\infty\}$ denotes the Brjuno function, a purely arithmetic $\mathbb{Z}$-periodic function. Its most important property is that $\Phi(\alpha)$ is finite if and only if $\alpha$ is a Brjuno number (see [MMY] for details on $\Phi)$.

The local conjugacy theorem, due to Yoccoz, states that any analytic circle map with a Brjuno rotation number and which is univalent in a sufficiently large annulus (where 'large' is defined only in terms of the rotation number) is analytically linearizable. Moreover, it gives a lower bound for the linearization domain which, again, only depends on the initial domain of univalency and the rotation number.

THEOREM 2.1. (Local conjugacy theorem) Let $\theta$ be a Brjuno number and $\Delta>0$ such that $\Delta>(1 / 2 \pi) \Phi(\theta)+C_{0}$, where $C_{0}$ is a universal constant. Let $f: \mathbb{T}^{1} \rightarrow \mathbb{T}^{1}$ be an analytic circle diffeomorphism, orientation preserving and with rotation number $\theta$. We assume that $f$ is holomorphic and univalent in the annulus $\mathcal{A}_{\Delta}$ (see (6)). Then, $f$ is analytically linearizable and the linearization $\eta: \mathbb{T}^{1} \rightarrow \mathbb{T}^{1}$ is analytic in the complex annulus $\mathcal{A}_{d}$, with

$$
d \geq \Delta-\frac{1}{2 \pi} \Phi(\theta)-C_{0}
$$

and verifies $\eta\left(\mathcal{A}_{d}\right) \subset \mathcal{A}_{\Delta}$.

Remark 2.2. For the Arnold standard family (1) a sort of reciprocal is also true. Indeed, it was shown in $[\mathbf{G}]$ that if a member of the Arnold standard family is analytically linearizable, then its rotation number must be Brjuno.

If $F$ is a holomorphic map leaving the unit circle invariant, then by applying Theorem 2.1 to the map $f$ induced by $F_{\mid \mathbf{C}_{1}}$, we can state an analogous result about the analytic linearization of $F$.

THEOREM 2.3. Let $\theta$ be a Brjuno number and $R>1$ such that $R>e^{\Phi(\theta)+2 \pi C_{0}}$. Let $F: \mathbf{C}_{1} \rightarrow \mathbf{C}_{1}$ be an analytic diffeomorphism with rotation number $\theta$. We assume that $F$ is holomorphic and univalent in the ring $A(1 / R, R)$. Then, $F$ is analytically linearizable and the linearization $\varphi: \mathbf{C}_{1} \rightarrow \mathbf{C}_{1}$ is analytic in the ring $A(1 / r, r)$, with

$$
r \geq R e^{-\Phi(\theta)-2 \pi C_{0}}
$$

and verifies $\varphi(A(1 / r, r)) \subset A(1 / R, R)$.

2.2. Quasiconformal mappings and the Beltrami equation. In this section we briefly recall the relevant definitions and results to be used in the quasiconformal surgery procedure, which is going to be one of the main tools to prove the results of this paper. 
The standard reference for quasiconformal mappings is $[\mathbf{A h}]$. In this section, $\mathcal{U}, \mathcal{V} \subset \mathbb{C}$ are open sets.

Definition 2.4. Given a measurable function $\mu: \mathcal{U} \rightarrow \mathbb{C}$, we say that $\mu$ is a $k$-Beltrami coefficient of $\mathcal{U}$ if $|\mu(z)| \leq k<1$ almost everywhere in $\mathcal{U}$. Two Beltrami coefficients of $\mathcal{U}$ are equivalent if they coincide almost everywhere in $\mathcal{U}$.

Equivalently, a Beltrami coefficient of $\mathcal{U}$ gives an almost complex structure $\sigma$, which means a measurable field of ellipses in the tangent space of $\mathcal{U}$, centered at 0 and defined up to multiplication by a non-zero real constant. The argument of the major axis of these infinitesimal ellipses, at the point $z \in \mathcal{U}$, is $\pi / 2+\arg (\mu(z)) / 2$, and the ratio of minor and major axes equals $(1-|\mu(z)|) /(1+|\mu(z)|)$.

Definition 2.5. A homeomorphism $f: \mathcal{U} \rightarrow \mathcal{V}$ is said to be $k$-quasiconformal if it has locally square integrable weak derivatives and

$$
\mu_{f}(z)=\frac{(\partial f / \partial \bar{z})(z)}{(\partial f / \partial z)(z)}
$$

is a $k$-Beltrami coefficient. In this case, we say that $\mu_{f}$ is the complex dilatation or the Beltrami coefficient of $f$.

Remark 2.6. With the same definition, but skipping the hypothesis on $f$ to be a homeomorphism, $f$ is called a $k$-quasiregular map. It is easy to check that a $k$-quasiregular map is locally the composition $g \circ h$ of a holomorphic map $g$ and a $k$-quasiconformal map $h$.

Definition 2.7. Given a Beltrami coefficient $\mu$ of $\mathcal{V}$ and a quasiregular map $h: \mathcal{U} \rightarrow \mathcal{V}$, we define the pull-back of $\mu$ by $h$ as the Beltrami coefficient of $\mathcal{U}$ defined by:

$$
h^{*} \mu=\frac{(\partial h / \partial \bar{z})+(\mu \circ h) \overline{(\partial h / \partial z)}}{(\partial h / \partial z)+(\mu \circ h) \overline{(\partial h / \partial \bar{z})}} .
$$

Remark 2.8. Note that if in the previous definition $\mu=\mu_{f}$ for certain quasiregular map $f$, then $h^{*} \mu_{f}=\mu_{f \circ h}$.

Remark 2.9. Pulling-back by holomorphic functions does not increase the maximal dilatation, $k$, of a $k$-Beltrami coefficient.

Remark 2.10. The standard complex structure corresponds to $\mu_{0} \equiv 0$, which is a field of circles. A quasiregular mapping $f$ is holomorphic if and only if $f^{*} \mu_{0}=\mu_{0}$.

Definition 2.11. Given a Beltrami coefficient $\mu$, the partial differential equation

$$
\frac{\partial f}{\partial \bar{z}}=\mu(z) \frac{\partial f}{\partial z}
$$

is called the Beltrami equation. By the integration of $\mu$ we mean the construction of a quasiconformal map $f$ solving this equation almost everywhere or, equivalently, such that $\mu_{f}=\mu$ almost everywhere.

The famous measurable Riemann mapping theorem by Morrey, Bojarski, Ahlfors and Bers states that every almost complex structure is integrable. As we are going to use this result for Beltrami coefficients with $\mathcal{U}=\mathcal{V}=\mathbb{C}$, we give a statement adapted to this context. 
THEOREM 2.12. (Measurable Riemann mapping theorem [Ah, BD]) Let $\mu$ be a Beltrami coefficient of $\mathbb{C}$. Then, there exists a unique quasiconformal map $h: \mathbb{C} \rightarrow \mathbb{C}$ such that $h(0)=0, h(1)=1$ and $\mu_{h}=\mu$. Furthermore, if $\mu_{t}$ is a family of Beltrami coefficients such that $\mu_{t}(z)$ depends analytically on $t$, for any $z \in \mathbb{C}$, then $h_{t}$ depends analytically on $t$.

Remark 2.13. The application of the measurable Riemann mapping theorem to complex dynamics is the following. Let $f$ and $\mu$ be, respectively, a quasiregular mapping of $\mathbb{C}$ and a Beltrami coefficient of $\mathbb{C}$, such that $f^{*} \mu=\mu$. If we apply Theorem 2.12 to integrate $\mu$ and we construct a quasiconformal mapping $h$ such that $\mu_{h}=\mu$, then $g=h \circ f \circ h^{-1}$ verifies $g^{*} \mu_{0}=\mu_{0}$, and hence $g$ is a holomorphic map of $\mathbb{C}$. Moreover, $f$ and $g$ are quasiconformally conjugate, i.e. they have the same dynamics.

2.3. Hyperbolic geometry. In this paper, besides quasiconformal surgery we will use some results of hyperbolic geometry (see [Be2] for a survey). Briefly, quasiconformal surgery will be the key for the geometrical constructions we do and hyperbolic geometry will provide some of the quantitative estimates.

Definition 2.14. Given $\mathcal{U} \subseteq \mathbb{C}$ a domain (open and connected set) and given a continuous function $\lambda: \mathcal{U} \rightarrow[0,+\infty)$, with at most isolated zeros, we define the conformal metric $\lambda$ on $\mathcal{U}$ as the metric having $\lambda(z)|d z|$ as a line element. More precisely, given a piecewise differentiable arc $\gamma:[a, b] \rightarrow \mathcal{U}$, the length of $\gamma$ with respect to the metric $\lambda$ is defined by

$$
l_{\lambda}(\gamma)=\int_{\gamma} \lambda(z)|d z|=\int_{a}^{b} \lambda(\gamma(t))\left|\gamma^{\prime}(t)\right| d t .
$$

Definition 2.15. Given a conformal metric $\lambda$ on $\mathcal{U}$ and given two points $z_{1}, z_{2} \in \mathcal{U}$, we define the distance $\mathrm{d}_{\lambda}\left(z_{1}, z_{2}\right)$ by

$$
\mathrm{d}_{\lambda}\left(z_{1}, z_{2}\right)=\inf \left\{l_{\lambda}(\gamma) \mid \gamma \subset U \text { arc from } z_{1} \text { to } z_{2}\right\} .
$$

In the case when this infimum is achieved by an $\operatorname{arc} \gamma^{*}$ from $z_{1}$ to $z_{2}$, this arc $\gamma^{*}$ is called a geodesic between $z_{1}$ and $z_{2}$.

Any holomorphic map between two domains $\mathcal{U}$ and $\mathcal{V}$ can be used to transport a conformal metric on $\mathcal{V}$ to a conformal metric on $\mathcal{U}$.

Definition 2.16. Given a holomorphic map $f: \mathcal{U} \rightarrow \mathcal{V}$ and a conformal metric $\lambda$ on $\mathcal{V}$, we define the pull-back of $\lambda$ by $f$ as the conformal metric on $\mathcal{U}$ given by

$$
f^{*} \lambda=(\lambda \circ f)\left|f^{\prime}\right|
$$

With this definition $f$ is a local isometry between $\left(\mathcal{U}, f^{*} \lambda\right)$ and $(\mathcal{V}, \lambda)$, i.e. it preserves arc-lengths. If $f$ is biholomorphic, then it is a global isometry.

The example that concerns us is the hyperbolic metric, which is a conformal metric defined on domains $\mathcal{U}$ that have the unit disk $\mathbb{D}:=\mathbb{D}_{1}$ as a covering space, and which is preserved under conformal self-mappings of $\mathcal{U}$. On $\mathbb{D}$, the hyperbolic metric takes the following form. 
Definition 2.17. The hyperbolic or Poincaré metric on $\mathbb{D}$ is the metric defined by

$$
\lambda_{\mathbb{D}}(z)=\frac{2}{1-|z|^{2}} .
$$

The Poincaré metric $\lambda_{\mathbb{D}}$ is the unique metric on $\mathbb{D}$ (up to multiplication by positive constants) invariant under conformal automorphisms of $\mathbb{D}$.

We will need an explicit expression for the distance in $\mathbb{D}$ defined by the Poincaré metric.

PROPOSITION 2.18. Given $w_{1}, w_{2} \in \mathbb{D}$, we have the following formula for the hyperbolic distance $\delta_{\mathbb{D}}$ in $\mathbb{D}$ :

$$
\sinh ^{2}\left[\frac{\delta_{\mathbb{D}}\left(w_{1}, w_{2}\right)}{2}\right]=\frac{4\left|w_{1}-w_{2}\right|^{2}}{\left(1-\left|w_{1}\right|^{2}\right)\left(1-\left|w_{2}\right|^{2}\right)}
$$

In particular, if $0 \leq r<1$,

$$
\delta_{\mathbb{D}}(0, r)=\log \left(\frac{1+r}{1-r}\right) .
$$

The pull-back process allows us to transport the Poincaré metric to any domain $\mathcal{U}$ that is conformally equivalent to $\mathbb{D}$. Indeed, if $\psi: \mathcal{U} \rightarrow \mathbb{D}$ is a Riemann map, then the hyperbolic metric on $\mathcal{U}$ is given by

$$
\lambda_{\mathcal{U}}(z)=\left(\psi^{*} \lambda_{\mathbb{D}}\right)(z)=\lambda_{\mathbb{D}}(\psi(z))\left|\psi^{\prime}(z)\right|,
$$

or equivalently, if $\varphi: \mathbb{D} \rightarrow \mathcal{U}$ is a conformal map, then

$$
\lambda_{\mathcal{U}}(\varphi(z))=\frac{\lambda_{\mathbb{D}}(z)}{\left|\varphi^{\prime}(z)\right|} .
$$

An important example of this is the upper half plane, $\mathbb{H}$, for which we can take $\psi(z)=$ $(z-i) /(z+i)$, obtaining the following result.

Proposition 2.19. The hyperbolic metric in $\mathbb{H}$ is given by $\lambda_{\mathbb{H}}(z)=1 / \operatorname{Im}(z)$. In this case, the $\lambda_{\mathbb{H}}$-geodesics are vertical segments or arcs of circles orthogonal to the real axis.

The hyperbolic metric can also be transported to non-simply connected domains, by means of any universal covering map.

Definition 2.20. A domain $\mathcal{U}$ of the Riemann sphere $\overline{\mathbb{C}}$ is called hyperbolic if it has at least three boundary points.

THEOREM 2.21. If $\mathcal{U}$ is a hyperbolic domain, there exists a holomorphic covering map $\varphi: \mathbb{D} \rightarrow \mathcal{U}$ (i.e. $\varphi$ is a local homeomorphism at every point). Each such map is called $a$ universal covering map and it is uniquely determined if we prefix $\varphi(0)$ and require $\varphi^{\prime}(0)>0$.

Then, if $\mathcal{U}$ is a hyperbolic domain and $\varphi$ is a universal covering for $\mathcal{U}$, the hyperbolic metric $\lambda_{\mathcal{U}}|d z|$ is given as above by (8).

Using that $\varphi(z)=\exp ((z-1) /(z+1))$ is a universal covering for the punctured disk $\mathbb{D}^{*}=\mathbb{D} \backslash\{0\}$, we obtain the following properties for the hyperbolic metric in $\mathbb{D}^{*}$. 
PROPOSITION 2.22. The hyperbolic metric in $\mathbb{D}^{*}$ has the form

$$
\lambda_{\mathbb{D}^{*}}(z)=\frac{1}{|z| \log (1 /|z|)} .
$$

The hyperbolic distance $\delta_{\mathbb{D}^{*}}$ satisfies

$$
\sinh ^{2}\left[\frac{\delta_{\mathbb{D}^{*}}\left(z_{1}, z_{2}\right)}{2}\right]=\frac{\left|\log z_{1}-\log z_{2}\right|^{2}}{\log \left|z_{1}\right| \log \left|z_{2}\right|}, \quad z_{1}, z_{2} \in \mathbb{D}^{*},
$$

where we have chosen appropriate determinations for $\log z_{1}$ and $\log z_{2}$ (with the arguments of $z_{1}$ and $z_{2}$ differing at most by $\pi$ ). Moreover, the geodesics in $\mathbb{D}^{*}$ are obtained by mapping the geodesics of $\mathbb{H}$ by the covering $\widehat{\varphi}: \mathbb{H} \rightarrow \mathbb{D}^{*}$ given by $\widehat{\varphi}(z)=e^{i z}$.

The main reason why hyperbolic geometry is very useful in complex dynamics is the fact that all holomorphic maps are contractive, when we look at them under the hyperbolic metric. This is known as the Schwarz-Pick lemma which reads as follows.

THEOREM 2.23. (Schwarz-Pick lemma) If $\mathcal{U}$ and $\mathcal{V}$ are hyperbolic domains and $f$ : $\mathcal{U} \rightarrow \mathcal{V}$ is holomorphic, then

$$
\delta \mathcal{V}\left(f\left(z_{1}\right), f\left(z_{2}\right)\right) \leq \delta_{\mathcal{U}}\left(z_{1}, z_{2}\right)
$$

for all $z_{1}, z_{2} \in \mathcal{U}$. Moreover, for all $z \in \mathcal{U}$,

$$
\frac{\lambda_{\mathcal{V}}(f(z))}{\lambda_{\mathcal{U}}(z)}\left|f^{\prime}(z)\right| \leq 1
$$

In this paper, we need to compare (locally) different hyperbolic distances. The following result, known as Ahlfors' lemma, gives a comparison between hyperbolic metrics. Since we are unable to provide a standard reference, we include its proof, taken from [Pet]. The analogous comparison for hyperbolic distances (which is in fact what we really need in the proof of Proposition B) requires some work and it is therefore given in Proposition 5.1 (see $§ 5.2)$.

Proposition 2.24. (Ahlfors' lemma) Let $\mathcal{U} \subseteq \mathcal{V} \subset \overline{\mathbb{C}}$ be hyperbolic domains. Then for any point $z \in \mathcal{U}$,

$$
1 \leq \frac{\lambda_{\mathcal{U}}(z)}{\lambda \mathcal{V}(z)} \leq \operatorname{coth}\left(\frac{1}{2} \delta \mathcal{V}(z, \partial \mathcal{U})\right)
$$

Proof. The left-hand inequality is quite immediate if we consider the identity map Id : $\mathcal{U} \rightarrow \mathcal{V}$. By the Schwarz-Pick lemma, $\lambda_{\mathcal{V}}(z) / \lambda_{\mathcal{U}}(z) \leq 1$ and we are done.

For the right-hand inequality, let $\varphi: \mathbb{D} \rightarrow \mathcal{V}$ be a universal covering of $\mathcal{V}$ such that $\varphi(0)=z$ and let $0 \in \mathcal{U}^{\prime} \subset \mathbb{D}$ be such that $\varphi: \mathcal{U}^{\prime} \rightarrow \mathcal{U}$ is conformal (see Figure 2).

Since $\varphi$ is a (local) isometry between the hyperbolic metrics, we may work with $\mathbb{D}, \mathcal{U}^{\prime}$ and 0 instead of $\mathcal{V}, \mathcal{U}$ and $z$. In particular, we have

$$
\frac{\lambda_{\mathcal{U}}(z)}{\lambda_{\mathcal{V}}(z)}=\frac{\lambda_{\mathcal{U}^{\prime}}(0)}{\lambda_{\mathbb{D}}(0)}
$$

Let $r=\min \left\{|z|: z \in \partial \mathcal{U}^{\prime}\right\}$. If $r=1$ then $\mathcal{U}^{\prime}=\mathbb{D}$ and there is nothing to prove. Hence we suppose $r<1$. We now apply the left-hand inequality to $\mathbb{D}_{r} \subseteq \mathcal{U}^{\prime}$ to obtain

$$
1 \leq \frac{\lambda_{\mathbb{D}_{r}}(0)}{\lambda \mathcal{U}^{\prime}(0)}=\frac{1}{r} \frac{\lambda \mathbb{D}(0)}{\lambda \mathcal{U}^{\prime}(0)},
$$




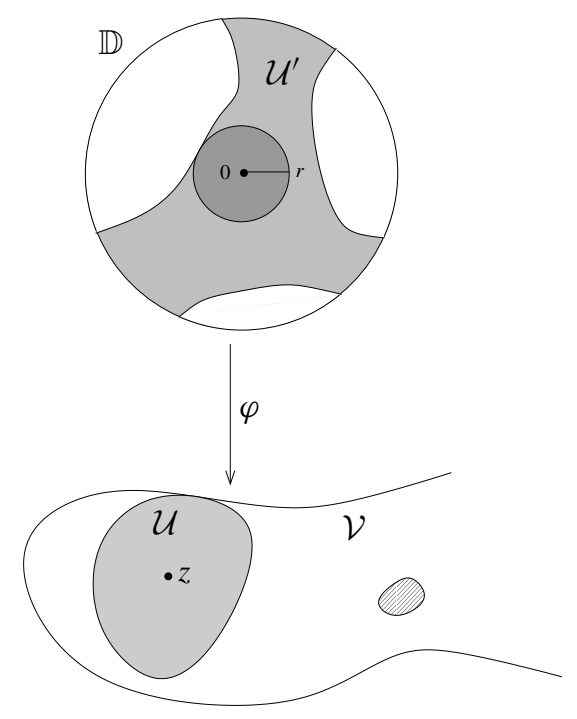

FIGURE 2. Sketch for the proof of Ahlfors' lemma.

and hence,

$$
\frac{\lambda_{\mathcal{U}}(z)}{\lambda_{\mathcal{V}}(z)} \leq \frac{1}{r}
$$

It remains to show that $1 / r=\operatorname{coth}(d / 2)$ where $d=\delta_{\mathcal{V}}(z, \partial \mathcal{U})$. To see this, we use Proposition 2.18. In particular, we observe that $\delta_{\mathbb{D}}(0, \cdot)$ has radial symmetry, and thus we have

$$
d=\delta_{\mathcal{V}}(z, \partial \mathcal{U})=\delta_{\mathbb{D}}\left(0, \partial \mathcal{U}^{\prime}\right)=\delta_{\mathbb{D}}(0, r)=\log \left(\frac{1+r}{1-r}\right) .
$$

Therefore $e^{d}=(1+r) /(1-r)$ and

$$
\frac{1}{r}=\frac{e^{d}+1}{e^{d}-1}=\frac{e^{d / 2}+e^{-d / 2}}{e^{d / 2}-e^{-d / 2}}=\frac{\cosh (d / 2)}{\sinh (d / 2)}=\operatorname{coth}\left(\frac{d}{2}\right) .
$$

3. The complex standard family and the semistandard map

The complex standard family

$$
\widetilde{F}_{\alpha, \varepsilon}(u)=u e^{i \alpha} e^{(\varepsilon / 2)(u-1 / u)},
$$

with $\alpha \in[0,2 \pi)$ and $\varepsilon \in[0,1)$, is a family of holomorphic maps of $\mathbb{C}^{*}$ onto itself, with essential singularities at 0 and infinity. The maps of the family are symmetric with respect to the unit circle, which is invariant under $\widetilde{F}_{\alpha, \varepsilon}$. The singularities of the inverse map consist exclusively of the images of the two critical points of $\widetilde{F}_{\alpha, \varepsilon}$ (as $\widetilde{F}_{\alpha, \varepsilon}$ has no asymptotic values) which are located at

$$
\widetilde{c}_{ \pm}(\varepsilon)=\frac{1}{\varepsilon}\left(-1 \pm \sqrt{1-\varepsilon^{2}}\right)<0 .
$$


Moreover, one can see that the standard map is univalent on a symmetric ring $A\left(1 / r_{\varepsilon}, r_{\varepsilon}\right)$, where

$$
r_{\varepsilon}=-\widetilde{c}_{-}(\varepsilon)=\frac{1}{\varepsilon}\left(1+\sqrt{1-\varepsilon^{2}}\right) .
$$

Note that $r_{\varepsilon} \lesssim 2 / \varepsilon$ as $\varepsilon \rightarrow 0$, and so $r_{\varepsilon}$ tends to infinity as $\varepsilon$ tends to zero.

From now on, we fix a rotation number $\theta$ in the Brjuno set and consider the analytic curve $\alpha=\alpha(\varepsilon)$ such that the rotation number of $\widetilde{F}_{\alpha(\varepsilon), \varepsilon \mid \mathbf{C}_{1}}$ is $\theta$. Thus, for $\varepsilon$ small enough (depending only on $\theta$ ), the standard map is under the hypothesis of the local conjugacy theorem of Yoccoz (Theorem 2.3) which assures the existence of a Herman ring of size

$$
\widetilde{R}_{\varepsilon} \geq \frac{1+\sqrt{1-\varepsilon^{2}}}{\varepsilon} K:=\frac{\sigma(\varepsilon)}{\varepsilon} K,
$$

where $K=\exp \left(-\Phi(\theta)-2 \pi C_{0}\right), \Phi$ is the Brjuno function and $C_{0}$ is a universal constant.

To asymptotically estimate the value of $\widetilde{R}_{\varepsilon}$, we start by scaling the problem hoping that the scaled value of $\widetilde{R}_{\varepsilon}$ has a finite limit as $\varepsilon$ tends to zero. We perform the change of variables

$$
z=\frac{\varepsilon}{2} u,
$$

and we obtain a new map

$$
F_{\alpha(\varepsilon), \varepsilon}(z)=z e^{i \alpha(\varepsilon)} e^{z-\varepsilon^{2} / 4 z} .
$$

This map shows the complex standard family as a perturbation of the semistandard map $G(z)=z e^{i \omega} e^{z}$, with $\omega=2 \pi \theta$, as long as $z$ is far away from zero (recall that $\alpha(0)=\omega$ ). Note that the limit is a singular limit at $z=0$, since an essential singularity is converted into a fixed point. The new scaled map $F_{\alpha(\varepsilon), \varepsilon}$ leaves $\mathbf{C}_{\varepsilon / 2}$ invariant and its critical points are now located at

$$
c_{ \pm}(\varepsilon)=\frac{1}{2}\left(-1 \pm \sqrt{1-\varepsilon^{2}}\right)<0
$$

which approach 0 and -1 as $\varepsilon$ tends to 0 .

We also change variables on the conjugation plane so that the map

$$
\varphi_{\varepsilon}(z)=\frac{\varepsilon}{2} \widetilde{\varphi}_{\varepsilon}\left(\frac{2}{\varepsilon} z\right),
$$

where $\widetilde{\varphi}_{\varepsilon}$ is given in (3), is now the linearizing map of $F_{\alpha(\varepsilon), \varepsilon \mid \mathbf{C}_{\frac{\varepsilon}{2}}}$. The map $\varphi_{\varepsilon}$ is defined from the ring $A\left(\varepsilon^{2} / 4 R_{\varepsilon}, R_{\varepsilon}\right)$, with $R_{\varepsilon}:=(\varepsilon / 2) \widetilde{R}_{\varepsilon}$, to the scaled Herman ring $U_{\varepsilon}:=\varepsilon / 2 \cdot \widetilde{U}_{\varepsilon}$ (see Figure 3).

We will actually compare the scaled standard family, $F_{\alpha(\varepsilon), \varepsilon}(z)$, with the semistandard map, $G(z)$. The qualitative and quantitative relationship between these maps will be explained by the surgery construction in the next section.

Remark 3.1. At this point, after scaling, Theorem A is equivalent to proving that

$$
R_{\varepsilon}=R_{0}+\mathcal{O}(\varepsilon \log \varepsilon)
$$

where $R_{0}$ is the conformal radius of the Siegel disk $U$ of the semistandard map $G(z)=$ $z e^{i \omega} e^{z}$ (see (5)). In particular, this result implies that $R_{\varepsilon}$ is a continuous function at $\varepsilon=0$.

Note that (12) means that Yoccoz's estimate (9) can be improved for the standard family by observing that $\widetilde{R}_{\varepsilon}=(\sigma(\varepsilon) / \varepsilon) K(\varepsilon)$, with $K(\varepsilon)=R_{0}+\mathcal{O}(\varepsilon \log \varepsilon)$ and, hence, $K(\varepsilon) \rightarrow R_{0}$ as $\varepsilon \rightarrow 0$. 


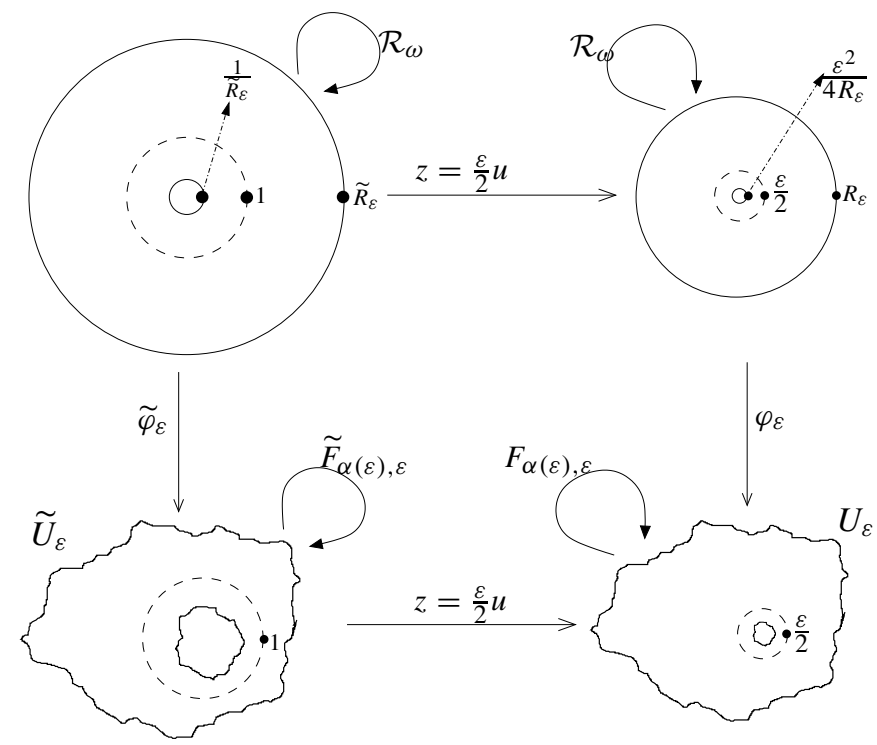

FIGURE 3. Scaling the dynamical plane and the linearizing plane.

\section{Proof of Theorem A}

The proof of Theorem A is based on an explicit (quantitative) version of the (qualitative) surgery construction $[\mathbf{G}]$ that relates a member of the (non-scaled) complex standard family $\widetilde{F}_{\alpha(\varepsilon), \varepsilon}$, with the semistandard map $G$. First, in $\$ 4.1$ we explain Geyer's construction, slightly modified and adapted to the scaled map $F_{\alpha(\varepsilon), \varepsilon}$. In $\$ 4.2$ we re-formulate Theorem A in terms of the previous surgery construction. Section 4.3 gives an explicit version of Geyer's construction, which allows us to obtain the quantitative results. In $\$ 4.4$ we obtain Theorem A as an easy consequence of Proposition B and the results of $\$ 4.3$. Finally, $\$ 5$ contains the proof of Proposition B.

4.1. Surgery construction. The idea of Geyer's construction to relate $F_{\alpha(\varepsilon), \varepsilon}$ to $G$ is basically to 'fill up the hole' of the Herman ring $U_{\varepsilon}$ in order to transform the Herman ring into a 'Siegel disk'. For our purposes, 'the hole' is the disk of radius $\varepsilon / 2$ (denoted by $\mathbb{D}_{\varepsilon / 2}$ ), given the fact that its boundary is simpler than the boundary of $U_{\varepsilon}$ and that it is invariant under the map. This can be accomplished by defining a new map $H_{\varepsilon}$ which consists of the old one $F_{\alpha(\varepsilon), \varepsilon}$ everywhere outside $\mathbb{D}_{\varepsilon / 2}$, and a suitable quasiconformal map conjugate to a rotation of angle $\omega$ inside this disk.

Due to the fact that the behaviour of the scaled standard map and the semistandard map at $\infty$ are the same, the map $H_{\varepsilon}$ thus obtained is then 'morally' equivalent (in the dynamical sense) to the semistandard map. However, the map $H_{\varepsilon}$ constructed in this way will be quasiregular, but not holomorphic. We shall make it holomorphic by means of the measurable Riemann mapping theorem (see Theorem 2.12), as explained in Remark 2.13. So, we construct a Beltrami coefficient $\mu_{\varepsilon}$, invariant by $H_{\varepsilon}$, and by the integration of $\mu_{\varepsilon}$ we will obtain a quasiconformal map $h_{\varepsilon}$ such that $h_{\varepsilon} \circ H_{\varepsilon} \circ h_{\varepsilon}^{-1}$ is holomorphic and has the 
same dynamics as $H_{\varepsilon}$ (holomorphic smoothing). By choosing $H_{\varepsilon}$ appropriately, we prove that this new map is the semistandard map $G$.

We now proceed to make this construction precise. To define a rotation inside the small disk $\mathbb{D}_{\varepsilon / 2}$, we first choose a 'gluing' map $\psi_{\varepsilon}$. Let $\psi_{\varepsilon}: \overline{\mathbb{D}_{\varepsilon / 2}} \rightarrow \overline{\mathbb{D}_{\varepsilon / 2}}$ be any quasiconformal map that agrees with $\varphi_{\varepsilon}$ on the boundary (i.e. $\psi_{\varepsilon \mid \mathbf{C}_{\varepsilon / 2}}=\varphi_{\varepsilon}$ ) and sends 0 to 0 . Since $\varphi_{\varepsilon}$ is a real analytic map, the existence of $\psi_{\varepsilon}$ is guaranteed (see [Pom]). Then we define the new map $H_{\varepsilon}$ as

$$
H_{\varepsilon}= \begin{cases}F_{\alpha(\varepsilon), \varepsilon} & \text { on } \mathbb{C} \backslash \mathbb{D}_{\varepsilon / 2} \\ \psi_{\varepsilon} \circ \mathcal{R}_{\omega} \circ \psi_{\varepsilon}^{-1} & \text { on } \overline{\mathbb{D}_{\varepsilon / 2}}\end{cases}
$$

(see Figure 4).

By the choice of $\psi_{\varepsilon}$, the map $H_{\varepsilon}$ is continuous and quasiregular. By construction, it has a fixed point at $z=0$, and it is conjugate to a rotation of angle $\omega$ on the set (topological disk) $\mathbb{D}_{\varepsilon / 2} \cup U_{\varepsilon}$ by means of the conjugacy maps $\varphi_{\varepsilon}$ and $\psi_{\varepsilon}$, which match up continuously. Last, we note that $H_{\varepsilon}$ has only one critical point: the former critical point $c_{-}(\varepsilon)$ of $F_{\alpha(\varepsilon), \varepsilon}$ (given in (10)) which was outside $\mathbb{D}_{\varepsilon / 2}$, since the symmetric one $c_{+}(\varepsilon)$ has been annihilated.

To start the second part of the surgery construction (holomorphic smoothing) we define the Beltrami coefficient $\mu_{\varepsilon}$ on $\mathbb{C}$ as follows. First we define it on the surgery region by pulling back $\mu_{0}=0$ to $\mathbb{D}_{\varepsilon / 2}$ by means of $\psi_{\varepsilon}^{-1}$. We extend this almost complex structure to every preimage of $\mathbb{D}_{\varepsilon / 2}$ using $H_{\varepsilon}$ (or equivalently $F_{\alpha(\varepsilon), \varepsilon}$, as both maps coincide outside of $\mathbb{D}_{\varepsilon / 2}$ ); and finally we set $\mu_{\varepsilon}=0$ at the remaining points. That is,

$$
\mu_{\varepsilon}= \begin{cases}\left(\psi_{\varepsilon}^{-1}\right)^{*}(0) & \text { on } \mathbb{D}_{\varepsilon / 2} \\ \left(H_{\varepsilon}^{n}\right)^{*}\left(\mu_{\varepsilon}\right) & \text { on } H_{\varepsilon}^{-n}\left(\mathbb{D}_{\varepsilon / 2}\right), \text { if } n \geq 1 \\ 0 & \text { on } \mathbb{C} \backslash \bigcup_{n \geq 0} H_{\varepsilon}^{-n}\left(\mathbb{D}_{\varepsilon / 2}\right),\end{cases}
$$

where $H_{\varepsilon}^{-n}\left(\mathbb{D}_{\varepsilon / 2}\right)$ should be understood as the set of points whose $n$th iterate falls (for the first time) in $\mathbb{D}_{\varepsilon / 2}$. Note that with this definition and using that $H_{\varepsilon}\left(U_{\varepsilon} \backslash \mathbb{D}_{\varepsilon / 2}\right)=U_{\varepsilon} \backslash \mathbb{D}_{\varepsilon / 2}$, we have that the points in $U_{\varepsilon} \backslash \mathbb{D}_{\varepsilon / 2}$ (and all their preimages) satisfy $\mu_{\varepsilon}(z)=0$. By construction, we have that $\mu_{\varepsilon}$ is measurable and invariant under the pull-back by $H_{\varepsilon}$, for it is spread out by the dynamics.

Remark 4.1. Since $\psi_{\varepsilon}$ is $k_{\varepsilon}$-quasiconformal in $\mathbb{D}_{\varepsilon / 2}$, for some $0<k_{\varepsilon}<1$, then $\mu_{\varepsilon}$ has maximal dilatation $\left\|\mu_{\varepsilon}\right\|_{\mathbb{D}_{\varepsilon / 2}}=\left\|\mu_{\psi_{\varepsilon}^{-1}}\right\|_{\mathbb{D}_{\varepsilon / 2}}=\left\|\mu_{\psi_{\varepsilon}}\right\|_{\mathbb{D}_{\varepsilon / 2}} \leq k_{\varepsilon}<1$, and also in the remainder of the plane since it is pulled-back by a holomorphic map (see Remark 2.9).

Therefore, we may apply the measurable Riemann mapping theorem (see Theorem 2.12) to $\tilde{\mu}_{\varepsilon}(z):=\mu_{\varepsilon}\left(c_{-}(\varepsilon) z\right)$, obtaining a (unique) quasiconformal mapping $\tilde{h}_{\varepsilon}: \mathbb{C} \rightarrow \mathbb{C}$ which integrates $\tilde{\mu}_{\varepsilon}$, that is $\mu_{\tilde{h}_{\varepsilon}}=\tilde{\mu}_{\varepsilon}$, and such that $\tilde{h}_{\varepsilon}(0)=0$ and $\widetilde{h}_{\varepsilon}(1)=1$. Now, we define $h_{\varepsilon}(z)=-\widetilde{h}_{\varepsilon}\left(z / c_{-}(\varepsilon)\right)$. We note that $h_{\varepsilon}$ integrates $\mu_{\varepsilon}$ and verifies $h_{\varepsilon}(0)=0$ and $h_{\varepsilon}\left(c_{-}(\varepsilon)\right)=-1$.

Remark 4.2. As $\mu_{\varepsilon}=0$ in $U_{\varepsilon} \backslash \overline{\mathbb{D}_{\varepsilon / 2}}$, we have from the Beltrami equation (7) that $h_{\varepsilon}$ is holomorphic in this region. 


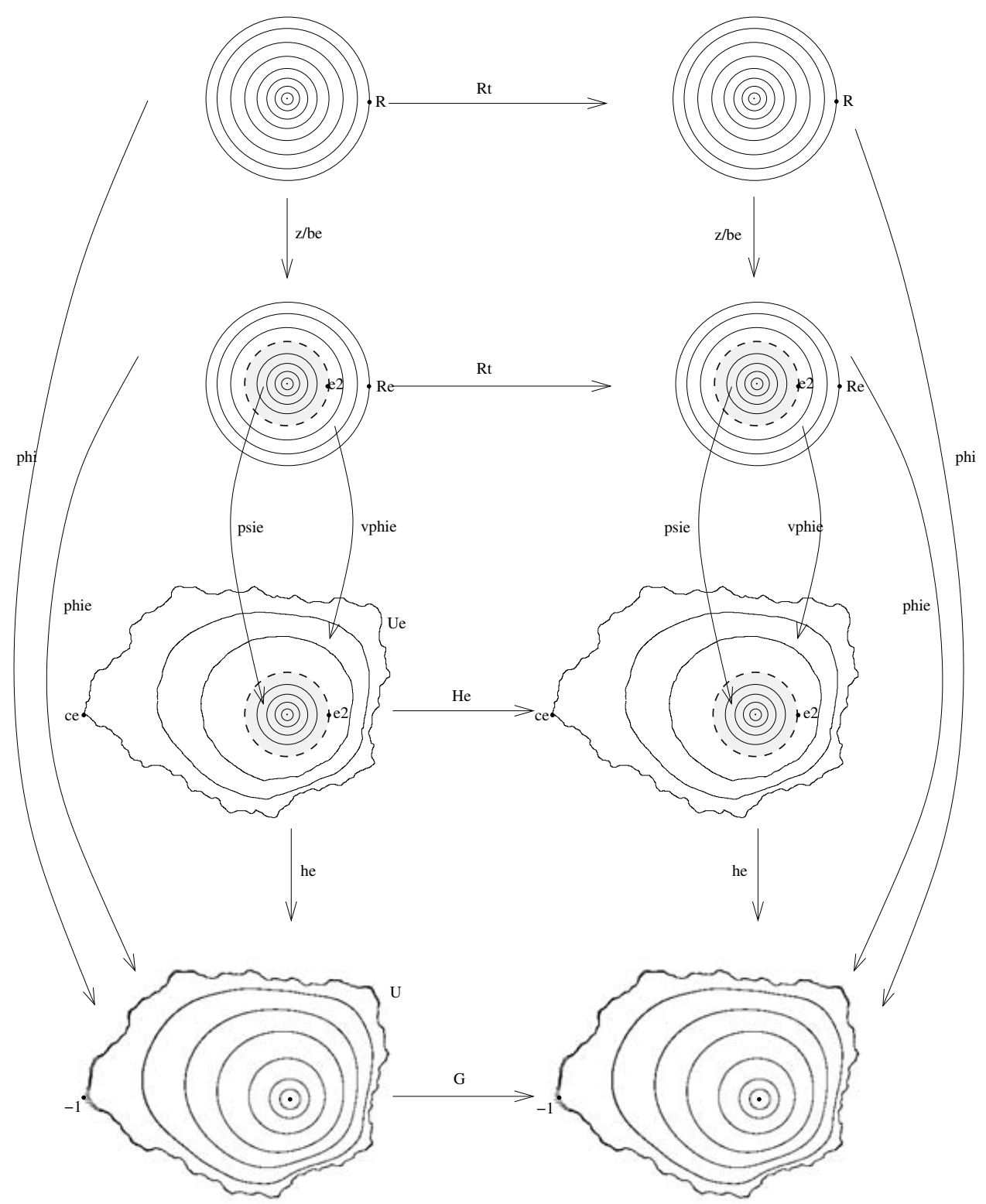

FIGURE 4. Commutative diagram showing all maps involved in the surgery construction. 
As explained in Remark 2.13, the composition map

$$
G_{\varepsilon}:=h_{\varepsilon} \circ H_{\varepsilon} \circ h_{\varepsilon}^{-1}
$$

is not only quasiconformally conjugate to $H_{\varepsilon}$ but also holomorphic in $\mathbb{C}$. Moreover, if $\left\|\mu_{\varepsilon}\right\|<\frac{1}{3}$, then we have that this map does not depend on the parameter $\varepsilon$ as shown in the following proposition.

PROPOSITION 4.3. With the previous notations, if $\left\|\mu_{\varepsilon}\right\|<\frac{1}{3}$, then for all $z \in \mathbb{C}$

$$
G_{\varepsilon}(z)=G(z)=z e^{i \omega} e^{z} .
$$

The proof of this proposition is similar to an analogous result for Geyer's construction in [G]. However, for the sake of completeness, we include here a proof adapted to our context. The key tool in the proof is an estimate on the growth of a quasiconformal map at $\infty$. This estimate is a consequence of the following basic property of quasiconformal maps.

THEOREM 4.4. [Ah, p. 71] A k-quasiconformal mapping in a domain $\mathcal{U} \subset \mathbb{C}$ is uniformly Hölder continuous with exponent $(1-k) /(1+k)$ in every compact subset of $\mathcal{U}$.

This result implies the desired bound for a quasiconformal map at $\infty$.

LEMMA 4.5. Let $\phi$ be a $k$-quasiconformal mapping of $\mathbb{C}$, fixing 0 and $\infty$. Then there exists $C>0$ such that for any $|z| \gg 1$ we have $|\phi(z)| \leq C|z|^{(1+k) /(1-k)}$.

Proof. We set $h=\phi^{-1}$ and $g(z)=1 / h(1 / z)$. It is easy to check that $g$ is $k$-quasiconformal, with $g(0)=0$ and $g(\infty)=\infty$. Applying Theorem 4.4 we have that there exists a constant $m>0$ such that

$$
\left|g\left(z_{1}\right)-g\left(z_{2}\right)\right| \leq m\left|z_{1}-z_{2}\right|^{(1-k) /(1+k)}, \quad \text { if }\left|z_{1}\right|,\left|z_{2}\right| \leq 1 .
$$

We take $z_{1}=z$ and $z_{2}=0$, and we replace $z=1 / \phi(w)$ to obtain the desired bound:

$$
|\phi(w)| \leq C|w|^{(1+k) /(1-k)},
$$

with $C=m^{(1+k) /(1-k)}$. However, this estimate holds provided that $|\phi(w)| \geq 1$. As $\{w \in \mathbb{C}:|\phi(w)| \leq 1\}$ is a compact set, we can assure that $|\phi(w)| \geq 1$ if $|w| \gg 1$.

Proof of Proposition 4.3. By construction, we know the following properties of $G_{\varepsilon}$ :

(a) $G_{\varepsilon}$ is entire;

(b) $G_{\varepsilon}(z)=0$ if and only if $z=0$;

(c) $G_{\varepsilon}$ has a Siegel disk $h_{\varepsilon}\left(\mathbb{D}_{\varepsilon / 2} \cup U_{\varepsilon}\right)$ around $z=0$, with rotation number $\theta$, and hence $G_{\varepsilon}^{\prime}(0)=e^{i \omega} ;$ and

(d) $G_{\varepsilon}^{\prime}(-1)=0$ because $G_{\varepsilon}$ is not univalent (it has degree two) around -1 .

Combining the first two properties of $G_{\varepsilon}$, we have that

$$
G_{\varepsilon}(z)=z g_{\varepsilon}(z)
$$

with $g_{\varepsilon}$ entire and without zeros. Now, we can estimate the growth order of $g_{\varepsilon}$. To this end, we use that if $z \in \mathbb{C} \backslash \mathbb{D}_{\varepsilon / 2}$, then

$$
H_{\varepsilon}(z)=F_{\alpha(\varepsilon), \varepsilon}(z)=z e^{i \alpha(\varepsilon)} e^{z-\varepsilon^{2} / 4 z} .
$$


So, we have that if $|z| \gg 1$, then

$$
G_{\varepsilon}(z)=h_{\varepsilon}\left(h_{\varepsilon}^{-1}(z) e^{i \alpha(\varepsilon)} e^{h_{\varepsilon}^{-1}(z)-\varepsilon^{2} / 4 h_{\varepsilon}^{-1}(z)}\right) .
$$

From Lemma 4.5 we have that if $|z| \gg 1$ there exists some constant $C>0$ such that $\left|h_{\varepsilon}(z)\right| \leq C|z|^{K_{\varepsilon}}$, where $K_{\varepsilon}=\left(1+\left\|\mu_{\varepsilon}\right\|\right) /\left(1-\left\|\mu_{\varepsilon}\right\|\right)$. For the other values of $z$ the map $h_{\varepsilon}$ is bounded. This is also true for $h_{\varepsilon}^{-1}$, and both facts can be summarized by saying that there exists $M>0$, which depends only on $\varepsilon$, such that

$$
\left|h_{\varepsilon}(z)\right| \leq M \max \left\{|z|^{K_{\varepsilon}}, 1\right\}, \quad\left|h_{\varepsilon}^{-1}(z)\right| \leq M \max \left\{|z|^{K_{\varepsilon}}, 1\right\} .
$$

Moreover, we may also ask that $\left|h_{\varepsilon}^{-1}(z)\right| \geq 1$ if $|z| \gg 1$, obtaining

$$
\left|G_{\varepsilon}(z)\right| \leq m_{1}|z|^{K_{\varepsilon}^{2}} e^{m_{2}|z|^{K_{\varepsilon}}},
$$

where $m_{1}$ and $m_{2}$ may depend on $\varepsilon$ (of course, the condition $|z| \gg 1$ is not necessarily uniform on $\varepsilon$ ). As we are assuming $\left\|\mu_{\varepsilon}\right\|<1 / 3$, we have that $1 \leq K_{\varepsilon}<2$, and so we deduce that $g_{\varepsilon}$ has growth order controlled by

$$
\left|g_{\varepsilon}(z)\right| \leq e^{|z|^{p}}, \quad \text { if }|z| \gg 1,
$$

with $1 \leq p<2$. The known properties of $g_{\varepsilon}(z)$ (entire function without zeros and with exponential growth of order $1 \leq p<2$ ) imply that it is of the form

$$
g_{\varepsilon}(z)=e^{P_{\varepsilon}(z)},
$$

(see [D]) with $P_{\varepsilon}(z)$ a polynomial of degree not greater than 1 . Now, the proposition follows from the remaining properties.

Remark 4.6. Assuming that $\left\|\mu_{\varepsilon}\right\|<\frac{1}{3}$, we have just proved that $G=h_{\varepsilon} \circ H_{\varepsilon} \circ h_{\varepsilon}^{-1}$, and so $H_{\varepsilon}$ and $G$ are conjugated by $h_{\varepsilon}$. Then, as the invariant curves are preserved by conjugation, we have that the rotation domain $\mathbb{D}_{\varepsilon / 2} \cup U_{\varepsilon}$ of $H_{\varepsilon}$ is mapped by $h_{\varepsilon}$ to the Siegel disk $U$ of $G$ (see (5)). That is

$$
U=h_{\varepsilon}\left(\mathbb{D}_{\varepsilon / 2} \cup U_{\varepsilon}\right) .
$$

This concludes the surgery construction relating the (scaled) standard map $F_{\alpha(\varepsilon), \varepsilon}$ and the semistandard map $G$. In the following section we see which quantities we need to estimate in order to obtain quantitative information from the surgery we just performed.

4.2. Restatement of the problem. Using the same notation as $\$ 4.1$ and assuming $\left\|\mu_{\varepsilon}\right\|$ $<\frac{1}{3}$, we observe (see Figure 4) that the map defined as

$$
\phi_{\varepsilon}(z)= \begin{cases}\left(h_{\varepsilon} \circ \varphi_{\varepsilon}\right)(z) & \text { if } z \in \mathbb{D}_{R_{\varepsilon} \backslash \mathbb{D}_{\varepsilon / 2}} \\ \left(h_{\varepsilon} \circ \psi_{\varepsilon}\right)(z) & \text { if } z \in \mathbb{D}_{\varepsilon / 2}\end{cases}
$$

is holomorphic in $\mathbb{D}_{R_{\varepsilon}}$. From Remark 4.2, this assertion is obviously true in $\mathbb{D}_{R_{\varepsilon}} \backslash \mathbb{D}_{\varepsilon / 2}$. To prove the analyticity of $\phi_{\varepsilon}$ in $\mathbb{D}_{\varepsilon / 2}$, we can check that $\phi_{\varepsilon}^{*} \mu_{0}=\mu_{0}$ (see Remark 2.10), which follows from the fact that $\mu_{\varepsilon}=\left(\psi^{-1}\right)^{*} \mu_{0}$ in $\mathbb{D}_{\varepsilon / 2}$.

By construction, $\phi_{\varepsilon}$ conjugates the semistandard map $G$ on the Siegel disk $U=$ $h_{\varepsilon}\left(\mathbb{D}_{\varepsilon / 2} \cup U_{\varepsilon}\right)$ to the rotation $\mathcal{R}_{\omega}$ on $\mathbb{D}_{R_{\varepsilon}}$. 
However, we cannot be sure that the map $\phi_{\varepsilon}$ is the normalized linearizing map $\varphi$ of $G$ (see (5)), since, among other reasons, we expect the radius $R_{\varepsilon}$ to move with $\varepsilon$ while $G$, $U$ and $R_{0}$ do not. This is equivalent to saying that $\phi_{\varepsilon}^{\prime}(0) \neq 1$. Then, to recuperate the (normalized) linearizing map $\varphi$, let us define

$$
b(\varepsilon)=\phi_{\varepsilon}^{\prime}(0),
$$

and so by the preceding argument,

$$
\varphi(z)=\phi_{\varepsilon}\left(\frac{z}{b(\varepsilon)}\right)
$$

given that $\phi_{\varepsilon}(z / b(\varepsilon))$ satisfies both normalization conditions $\left(\varphi(0)=0, \varphi^{\prime}(0)=1\right)$ (see Figure 4).

From here, it is clear that $R_{\varepsilon}=R_{0} /|b(\varepsilon)|$, so that to relate $R_{0}$ and $R_{\varepsilon}$ we need to have control over $b(\varepsilon)$, i.e. over $\left(h_{\varepsilon} \circ \psi_{\varepsilon}\right)^{\prime}(0)$.

Remark 4.7. Let us observe that $\left(h_{\varepsilon} \circ \psi_{\varepsilon}\right)^{\prime}(0)$ does not depend on the particular quasiconformal map $\psi_{\varepsilon}$ used in the surgery construction (which is of course not unique). This allows us to compute this derivative by explicitly constructing a convenient $\psi_{\varepsilon}$.

From the previous observations, Theorem A follows immediately from the next proposition.

PROPOSITION 4.8. With the previous notation, we have

$$
b(\varepsilon)=\left(h_{\varepsilon} \circ \psi_{\varepsilon}\right)^{\prime}(0)=1+\mathcal{O}(\varepsilon \log \varepsilon) .
$$

To prove Proposition 4.8, we study the quantity

$$
\frac{d}{d z}\left(h_{\varepsilon} \circ \psi_{\varepsilon}(z)-z\right)_{\mid z=0}
$$

by means of the Cauchy integral formula. This will be done in $\$ 4.4$. The estimates we use come from studying the quantities $\left|\psi_{\varepsilon}(z)-z\right|$ and $\left|h_{\varepsilon}(z)-z\right|$ or, equivalently, how far the maps $\psi_{\varepsilon}$ and $h_{\varepsilon}$ are from the identity map in a neighborhood of zero.

To obtain such an estimate for $\psi_{\varepsilon}$ we construct $\psi_{\varepsilon}$ explicitly in $\S 4.3$. The estimate for $h_{\varepsilon}$ is a direct application of Proposition B.

4.3. Explicit surgery construction. The main purpose of this section is to explicitly construct the quasiconformal extension $\psi_{\varepsilon}$ used in the surgery construction of $\S 4.1$, and to give the explicit estimates that measure how far $\psi_{\varepsilon}$ is from the identity map.

Let us recall that we have a circle of radius $\varepsilon / 2$ on which the real analytic (scaled) conjugacy $\varphi_{\varepsilon}$ is defined. Our goal is to find a quasi-conformal map $\psi_{\varepsilon}: \mathbb{D}_{\varepsilon / 2} \rightarrow \mathbb{D}_{\varepsilon / 2}$ that extends $\varphi_{\varepsilon}$.

We define the 'gluing map' $\psi_{\varepsilon}$ to be the most natural extension: the radial one. More explicitly, given $z \in \mathbb{D}_{\varepsilon / 2}$ we define

$$
\psi_{\varepsilon}(z)=\frac{2}{\varepsilon}|z| \varphi_{\varepsilon}\left(\frac{\varepsilon}{2} \frac{z}{|z|}\right) .
$$


This map is clearly continuous, it agrees with $\varphi_{\varepsilon}$ on the boundary of $\mathbb{D}_{\varepsilon / 2}$ and sends 0 to 0 . We also observe that it leaves all circles in $\mathbb{D}_{\varepsilon / 2}$ invariant. In Proposition 4.9 below, we prove that $\psi_{\varepsilon}$ is a quasiconformal mapping if $\varepsilon$ is small enough (even more, it is $\mathcal{C}^{\infty}$ at all points except at $z=0$ ). Moreover, this result shows that for small values of $\varepsilon$ we have $\left\|\mu_{\varepsilon}\right\|<\frac{1}{3}$ (this condition is needed to show that $G_{\varepsilon}=G$, as stated in Proposition 4.3), and so all the results derived from the quasiconformal construction (see $\S \S 4.1$ and 4.2 ) hold.

Our goal in the remainder of this section is to prove the following result.

PROPOSITION 4.9. There exists a constant $C_{1}>0$, independent of $\varepsilon$, such that for any $\varepsilon$ small enough, $\psi_{\varepsilon}$ is a $\left(C_{1} \varepsilon\right)$-quasiconformal mapping in $\mathbb{D}_{\varepsilon / 2}$, and it verifies

$$
\left|\psi_{\varepsilon}(z)-z\right| \leq C_{1} \varepsilon|z|
$$

Remark 4.10. One could also use an alternative, more dynamically meaningful, quasiconformal extension of $\varphi_{\varepsilon}$ given by $\psi_{\varepsilon}(z)=\varphi_{2|z|}(z)$. In this case, the map $H_{\varepsilon}$ would be explicitly given by $H_{\varepsilon}(z)=F_{\alpha(2|z|), 2|z|}(z)=z e^{i \alpha(2|z|)} e^{z-\bar{z}}$ on $\mathbb{D}_{\varepsilon / 2}$. This extension also preserves circles in $\mathbb{D}_{\varepsilon / 2}$ and, on each of these circles, it is the linearizing map of a scaled standard map. In this case, one can show that $\left|\psi_{\varepsilon}(z)-z\right| \leq C_{1}|z|^{2}$.

The proof of this proposition will be an easy consequence of the following lemma.

LEMMA 4.11. The linearization $\varphi_{\varepsilon}$ (see (11)) of the scaled standard map $F_{\alpha(\varepsilon), \varepsilon}(z)=$ $z e^{i \alpha(\varepsilon)} e^{z-\varepsilon^{2} / 4 z}$, verifies the following bounds if $\varepsilon$ is small enough and $|z|=\varepsilon / 2$ :

$$
\left|\varphi_{\varepsilon}(z)-z\right| \leq C_{2} \varepsilon^{2}, \quad\left|\varphi_{\varepsilon}^{\prime}(z)-1\right| \leq C_{2} \varepsilon
$$

where $C_{2}$ is a constant independent of $\varepsilon$.

The proof of Lemma 4.11 is deferred to the end of the section.

Proof of Proposition 4.9. First of all, we stress that the estimates given by Lemma 4.11 are only valid if we evaluate $\varphi_{\varepsilon}(z)$ for $|z|=\varepsilon / 2$. From the definition of $\psi_{\varepsilon}(z)$ (see (13)) this is precisely the case which we are interested in.

Let us see that $\psi_{\varepsilon}$ is a quasiconformal mapping, and obtain a bound for its distortion. In $\mathbb{D}_{\varepsilon / 2}$ we have

$$
\frac{\partial \psi_{\varepsilon}}{\partial z}(z)=\frac{1}{2} \varphi_{\varepsilon}^{\prime}\left(\frac{\varepsilon}{2} \frac{z}{|z|}\right)+\frac{1}{\varepsilon} \frac{\bar{z}}{|z|} \varphi_{\varepsilon}\left(\frac{\varepsilon}{2} \frac{z}{|z|}\right)
$$

and

$$
\frac{\partial \psi_{\varepsilon}}{\partial \bar{z}}(z)=-\frac{1}{2} \frac{z^{2}}{|z|^{2}} \varphi_{\varepsilon}^{\prime}\left(\frac{\varepsilon}{2} \frac{z}{|z|}\right)+\frac{1}{\varepsilon} \frac{z}{|z|} \varphi_{\varepsilon}\left(\frac{\varepsilon}{2} \frac{z}{|z|}\right) .
$$

Then, applying Lemma 4.11, we can bound

$$
\begin{aligned}
\left|\frac{\partial \psi_{\varepsilon}}{\partial z}(z)-1\right| & =\left|\frac{1}{2}\left(\varphi_{\varepsilon}^{\prime}\left(\frac{\varepsilon}{2} \frac{z}{|z|}\right)-1\right)+\frac{1}{\varepsilon} \frac{\bar{z}}{|z|}\left(\varphi_{\varepsilon}\left(\frac{\varepsilon}{2} \frac{z}{|z|}\right)-\frac{\varepsilon}{2} \frac{z}{|z|}\right)\right| \\
& \leq \frac{1}{2} C_{2} \varepsilon+\frac{1}{\varepsilon} C_{2} \varepsilon^{2} \leq \frac{3}{2} C_{2} \varepsilon
\end{aligned}
$$


and

$$
\begin{aligned}
\left|\frac{\partial \psi_{\varepsilon}}{\partial \bar{z}}(z)\right| & =\left|-\frac{1}{2} \frac{z^{2}}{|z|^{2}}\left(\varphi_{\varepsilon}^{\prime}\left(\frac{\varepsilon}{2} \frac{z}{|z|}\right)-1\right)+\frac{1}{\varepsilon} \frac{z}{|z|}\left(\varphi_{\varepsilon}\left(\frac{\varepsilon}{2} \frac{z}{|z|}\right)-\frac{\varepsilon}{2} \frac{z}{|z|}\right)\right| \\
& \leq \frac{1}{2} C_{2} \varepsilon+\frac{1}{\varepsilon} C_{2} \varepsilon^{2} \leq \frac{3}{2} C_{2} \varepsilon
\end{aligned}
$$

So, if we assume $\varepsilon$ to be small enough in order to have that $\frac{3}{2} C_{2} \varepsilon \leq \frac{1}{2}$, we can bound the distortion of $\psi_{\varepsilon}$ by

$$
\left|\frac{\left(\partial \psi_{\varepsilon} / \partial \bar{z}\right)(z)}{\left(\partial \psi_{\varepsilon}\right) /(\partial z)(z)}\right| \leq \frac{(3 / 2) C_{2} \varepsilon}{1-(3 / 2) C_{2} \varepsilon} \leq 3 C_{2} \varepsilon
$$

Hence, $\psi_{\varepsilon}$ is a $\left(C_{1} \varepsilon\right)$-quasiconformal mapping with $C_{1}=3 C_{2}$.

In order to estimate how far $\psi_{\varepsilon}$ is from the identity map, we apply the first inequality of (15) (see Lemma 4.11), obtaining

$$
\left|\psi_{\varepsilon}(z)-z\right|=\left|\frac{2}{\varepsilon}\right| z\left|\left(\varphi_{\varepsilon}\left(\frac{\varepsilon}{2} \frac{z}{|z|}\right)-\frac{\varepsilon}{2} \frac{z}{|z|}\right)\right| \leq \frac{2}{\varepsilon}|z| C_{2} \varepsilon^{2} \leq C_{1} \varepsilon|z|
$$

which concludes the proof.

The rest of the section is dedicated to proving Lemma 4.11. For this purpose, we will need a diophantine-like bound for Brjuno numbers that is weaker than the Brjuno condition. More precisely, if $\theta$ is a Brjuno number, there exist constants $c_{1}, c_{2}$, depending only on $\theta$, such that for any $k \in \mathbb{Z} \backslash\{0\}$, the following inequality is satisfied (see [Br1, p. 140]):

$$
\left|e^{2 \pi i k \theta}-1\right|^{-1} \leq c_{1} e^{2 \pi c_{2}|k|}
$$

this inequality leads to the following lemma.

LEMMA 4.12. Let $m(x)$ be a 1-periodic function with zero average, and $\theta \in \mathbb{R}$ be a Brjuno number, hence verifying (16). We assume that $m$ is analytic in the complex annulus $\mathcal{A}_{c_{3}}=\left\{x \in \mathbb{C} / \mathbb{Z}:|\operatorname{Im}(x)|<c_{3}\right\}$, being $c_{3}>c_{2}$, and that $B=\sup _{x \in \mathcal{A}_{c_{3}}}|m(x)|<+\infty$.

Consider the 1-periodic solution $\xi(x)$ of the difference equation:

$$
\xi(x+\theta)-\xi(x)=m(x), \quad \xi(0)=0 .
$$

Then, $\xi$ is analytic in $\mathcal{A}_{c_{3}-c_{2}}$, and verifies

$$
|\xi(x)| \leq 4 B c_{1} \frac{e^{-2 \pi\left(c_{3}-c_{2}-|\operatorname{Im}(x)|\right)}}{1-e^{-2 \pi\left(c_{3}-c_{2}-|\operatorname{Im}(x)|\right)}}, \quad x \in \mathcal{A}_{c_{3}-c_{2}} .
$$

Proof. First, we expand $m$ in Fourier series:

$$
m(x)=\sum_{k \in \mathbb{Z}} m_{k} e^{2 \pi i k x} .
$$

Then, using the bound of $|m|$ in the annulus $\mathcal{A}_{c_{3}}$, and that it has zero average, one has that its Fourier coefficients verify $m_{0}=0$ and $\left|m_{k}\right| \leq B e^{-2 \pi c_{3}|k|}$ if $k \neq 0$. On the other hand, writing $\xi$ also in Fourier series, we can solve (17) for the coefficients of $\xi$, obtaining

$$
\xi_{k}=\frac{m_{k}}{e^{2 \pi i k \theta}-1}, \quad \text { if } k \neq 0
$$

Moreover, condition $\xi(0)=0$ gives $\xi_{0}=-\sum_{k \in \mathbb{Z} \backslash\{0\}} \xi_{k}$. 
Using (16) we obtain that

$$
\left|\xi_{k}\right| \leq c_{1}\left|m_{k}\right| e^{2 \pi c_{2}|k|} \leq B c_{1} e^{-2 \pi\left(c_{3}-c_{2}\right)|k|}, \quad \text { if } k \neq 0
$$

and, for $\xi_{0}$,

$$
\left|\xi_{0}\right| \leq \sum_{k \in \mathbb{Z} \backslash\{0\}}\left|\xi_{k}\right| \leq 2 B c_{1} \frac{e^{-2 \pi\left(c_{3}-c_{2}\right)}}{1-e^{-2 \pi\left(c_{3}-c_{2}\right)}}
$$

Joining these bounds, the estimate for $|\xi(x)|$ in $\mathcal{A}_{c_{3}-c_{2}}$ follows immediately.

Now, we have all the ingredients to prove Lemma 4.11.

Proof of Lemma 4.11. To obtain the estimates for this lemma, it will be more convenient for us to work with the map on the circle induced by the scaled standard map, that is the Arnold standard family (1). To this end, we make the transformations we have done backwards in order to go from $\widetilde{f}_{\alpha(\varepsilon), \varepsilon}(x)=x+\alpha(\varepsilon) / 2 \pi+(\varepsilon / 2 \pi) \sin (2 \pi x)$ to $F_{\alpha(\varepsilon), \varepsilon}(z)$. Then, if we make the change $z=(\varepsilon / 2) u$, we have $\widetilde{\varphi}_{\varepsilon}(u)=(2 / \varepsilon) \varphi_{\varepsilon}((\varepsilon / 2) u)$, where $\widetilde{\varphi}_{\varepsilon}$ is the linearization of the standard map $\widetilde{F}_{\alpha(\varepsilon), \varepsilon}$ given in (3). On the other hand, writing $u=e^{2 \pi i x}$ we have $\widetilde{\varphi}_{\varepsilon}\left(e^{2 \pi i x}\right)=e^{2 \pi i \widetilde{\eta}_{\varepsilon}(x)}, \widetilde{\eta}_{\varepsilon}$ being a conjugation of $\widetilde{f}_{\alpha(\varepsilon), \varepsilon}$ to the rotation $\mathcal{T}_{\theta}$ in $\mathbb{T}^{1}$ (see (2)).

Then, we obtain that

$$
\varphi_{\varepsilon}(z)-z=\frac{\varepsilon}{2}\left(\widetilde{\varphi}_{\varepsilon}(u)-u\right)=\frac{\varepsilon}{2}\left(e^{2 \pi i \widetilde{\eta}_{\varepsilon}(x)}-e^{2 \pi i x}\right)=z\left(e^{2 \pi i\left(\widetilde{\eta}_{\varepsilon}(x)-x\right)}-1\right) .
$$

Our purpose is to bound $\widetilde{\eta}_{\varepsilon}(x)-x$ using the local conjugacy Theorem 2.1, and to derive from these bounds those for $\varphi_{\varepsilon}(z)-z$ and its derivative.

First of all, we observe that $\widetilde{f}_{\alpha(\varepsilon), \varepsilon}(x)$ is univalent in the annulus $\mathcal{A}_{(1 / 2 \pi) \log (\sigma(\varepsilon) / \varepsilon)}$, where $\sigma(\varepsilon)=1+\sqrt{1-\varepsilon^{2}}$ is defined in (9).

In order to apply Theorem 2.1, let us observe that, if $\varepsilon$ is small enough, we have that $(1 / 2 \pi) \log (\sigma(\varepsilon) / \varepsilon)>(1 / 2 \pi) \Phi(\theta)+C_{0}$. Thus, Theorem 2.1 ensures that $\tilde{\eta}_{\varepsilon}(x)$ is analytic if

$$
|\operatorname{Im}(x)|<\frac{1}{2 \pi} \log (\sigma(\varepsilon) / \varepsilon)-\frac{1}{2 \pi} \Phi(\theta)-C_{0} .
$$

In particular, if we take any constant $0<c_{4} \leq \sigma(\varepsilon) / e^{\Phi(\theta)+2 \pi C_{0}}$, the linearization $\tilde{\eta}$ is

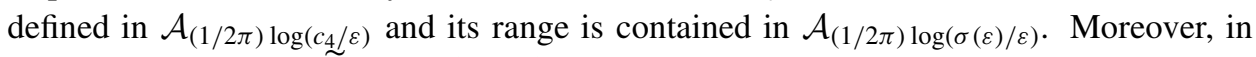
this domain it verifies $\widetilde{f}_{\alpha(\varepsilon), \varepsilon} \circ \widetilde{\eta}_{\varepsilon}=\widetilde{\eta}_{\varepsilon} \circ \mathcal{T}_{\theta}$.

Now, calling $\xi(x)=\tilde{\eta}_{\varepsilon}(x)-x$, it is straightforward to check that

$$
\xi(x+\theta)-\xi(x)=m(x)
$$

where $m(x)=\tilde{f}_{\alpha(\varepsilon), \varepsilon}\left(\tilde{\eta}_{\varepsilon}(x)\right)-\tilde{\eta}_{\varepsilon}(x)-\theta$. Then, $\xi(x)$ verifies the hypotheses of Lemma 4.12 if we define $c_{3} \equiv c_{3}(\varepsilon)=(1 / 2 \pi) \log \left(c_{4} / \varepsilon\right)$ and we take $B$ a bound of

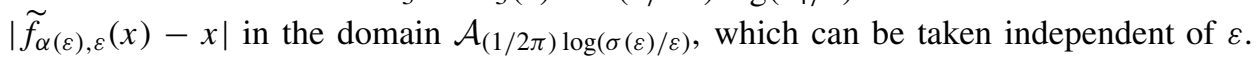
We remark that as $c_{3}=(1 / 2 \pi) \log \left(c_{4} / \varepsilon\right)$, the hypothesis $c_{3}>c_{2}$ of Lemma 4.12 always holds if we assume $\varepsilon<c_{4} e^{-2 \pi c_{2}}$.

Applying the conclusions of Lemma 4.12, we obtain the following bound for the function $\xi(x)$ when $|\operatorname{Im}(x)| \leq c_{3}-c_{2}=(1 / 2 \pi) \log \left(c_{4} / \varepsilon\right)-c_{2}$,

$$
|\xi(x)| \leq 4 B c_{1} \frac{e^{-\left(\log \left(c_{4} / \varepsilon\right)-2 \pi\left(c_{2}+|\operatorname{Im}(x)|\right)\right)}}{1-e^{-\left(\log \left(c_{4} / \varepsilon\right)-2 \pi\left(c_{2}+|\operatorname{Im}(x)|\right)\right)}}=\frac{4 B c_{1} \varepsilon}{c_{4} e^{-2 \pi\left(c_{2}+|\operatorname{Im}(x)|\right)}-\varepsilon} .
$$


Let us now take $c_{5}$ any constant, independent of $\varepsilon$, verifying $0<c_{5}<$ $(1 / 2 \pi) \log \left(c_{4} / 2 \varepsilon\right)-c_{2}$. We observe that $c_{5}$ can be taken arbitrarily large, provided that $\varepsilon$ is sufficiently small and that, with this definition of $c_{5}$, we always have the inequality $\varepsilon \leq\left(c_{4} / 2\right) e^{-2 \pi\left(c_{2}+c_{5}\right)}$.

Then, the previous considerations imply that the function $\tilde{\eta}_{\varepsilon}(x)-x=\xi(x)$ is defined in $\mathcal{A}_{c 5}$ and verifies, in this domain, the bound

$$
\left|\tilde{\eta}_{\varepsilon}(x)-x\right| \leq \frac{4 B c_{1} \varepsilon}{c_{4} e^{-2 \pi\left(c_{2}+c_{5}\right)}-\varepsilon} \leq \frac{8 B c_{1} e^{2 \pi\left(c_{2}+c_{5}\right)}}{c_{4}} \varepsilon .
$$

Using the changes $u=e^{2 \pi i x}$ and $z=(\varepsilon / 2) u$, we have that if $x \in \mathcal{A}_{c_{5}}$ then $u \in$ $A\left(e^{-2 \pi c_{5}}, e^{2 \pi c_{5}}\right)$ and $z \in A\left((\varepsilon / 2) e^{-2 \pi c_{5}},(\varepsilon / 2) e^{2 \pi c_{5}}\right)$ (see (4)). Now, we assume $\varepsilon$ small enough such that $\left(8 B c_{1} e^{2 \pi\left(c_{2}+c_{5}\right)} / c_{4}\right) \varepsilon \leq 1$. So, Equation (19) implies $\left|\widetilde{\eta}_{\varepsilon}(x)-x\right| \leq 1$. Then, for these values of $z$, inequality (18) leads to

$$
\left|\varphi_{\varepsilon}(z)-z\right| \leq|z| e^{2 \pi\left|\widetilde{\eta}_{\varepsilon}(x)-x\right|}\left|\widetilde{\eta}_{\varepsilon}(x)-x\right| \leq \frac{16 \pi B c_{1} e^{2 \pi\left(c_{2}+c_{5}+1\right)}}{c_{4}} \varepsilon|z|
$$

So, the first bound in (15) follows from (20) as a particular case when $|z|=\varepsilon / 2$, taking $C_{2} \geq 8 \pi B c_{1} e^{2 \pi\left(c_{2}+c_{5}+1\right)} / c_{4}$.

Using the bound of the function $\varphi_{\varepsilon}(z)-z$ in the ring $A\left((\varepsilon / 2) e^{-2 \pi c_{5}},(\varepsilon / 2) e^{2 \pi c_{5}}\right)$, we proceed to bound its derivative for $|z|=\varepsilon / 2$. To this end, we pick a particular value of $z$ and we consider the disk of center $z$ and radius $r=(\varepsilon / 2)\left(1-e^{-2 \pi c_{5}}\right)$, which we denote by $\mathbf{C}_{r}(z)$. One can easily check that if we take a point $t \in \mathbf{C}_{r}(z)$, then $t$ belongs to $A\left((\varepsilon / 2) e^{-2 \pi c_{5}},(\varepsilon / 2) e^{2 \pi c_{5}}\right)$ and then $\left|\varphi_{\varepsilon}(t)-t\right|$ verifies $(20)$ with $z \equiv t$.

Then, we can bound the derivative of $\varphi_{\varepsilon}(z)-z$ using the Cauchy integral formula. We have

$$
\varphi_{\varepsilon}^{\prime}(z)-1=\frac{d}{d t}\left(\varphi_{\varepsilon}(t)-t\right)_{\mid t=z}=\frac{1}{2 \pi i} \int_{\mathbf{C}_{r}(z)} \frac{\varphi_{\varepsilon}(t)-t}{(t-z)^{2}} d t
$$

In order to bound the integral above, we may apply inequality (20) to any $t \in \mathbf{C}_{r}(z)$, obtaining

$$
\begin{aligned}
\left|\varphi_{\varepsilon}^{\prime}(z)-1\right| & \leq \frac{1}{r} \max _{t \in \mathbf{C}_{r}(z)}\left|\varphi_{\varepsilon}(t)-t\right| \leq \frac{1}{r} \max _{t \in \mathbf{C}_{r}(z)}\left\{\frac{16 \pi B c_{1} e^{2 \pi\left(c_{2}+c_{5}+1\right)}}{c_{4}} \varepsilon|t|\right\} \\
& \leq \frac{2-e^{-2 \pi c_{5}}}{1-e^{-2 \pi c_{5}}} \frac{16 \pi B c_{1} e^{2 \pi\left(c_{2}+c_{5}+1\right)}}{c_{4}} \varepsilon,
\end{aligned}
$$

which gives the second part of (15) taking $C_{2} \geq\left(2-e^{-2 \pi c_{5}}\right) /\left(1-e^{-2 \pi c_{5}}\right)$ $\left(16 \pi B c_{1} e^{2 \pi\left(c_{2}+c_{5}+1\right)} / c_{4}\right)$.

4.4. Proof of Theorem A. From the restatement of the problem in $\$ 4.1$, we only need to prove Proposition 4.8 in order to prove Theorem A. 
Proof of Proposition 4.8. As we know that $h_{\varepsilon} \circ \psi_{\varepsilon}$ is holomorphic, we can express $b(\varepsilon)-1$ in terms of the Cauchy integral formula:

$$
\begin{aligned}
b(\varepsilon)-1 & =\frac{d}{d z}\left(h_{\varepsilon} \circ \psi_{\varepsilon}(z)-z\right)_{\mid z=0} \\
& =\frac{1}{2 \pi i} \int_{\mathbf{C}_{r}} \frac{h_{\varepsilon} \circ \psi_{\varepsilon}(z)-z}{z^{2}} d z \\
& =\frac{1}{2 \pi i} \int_{\mathbf{C}_{r}} \frac{h_{\varepsilon} \circ \psi_{\varepsilon}(z)-\psi_{\varepsilon}(z)}{z^{2}} d z+\frac{1}{2 \pi i} \int_{\mathbf{C}_{r}} \frac{\psi_{\varepsilon}(z)-z}{z^{2}} d z,
\end{aligned}
$$

where $\mathbf{C}_{r}$ is any circle contained in $\overline{\mathbb{D}_{\varepsilon / 2}}$. From now on we take $r=\varepsilon / 2$.

We can bound the second integral by using inequality (14) of Proposition 4.9, obtaining

$$
\left|\frac{1}{2 \pi i} \int_{\mathbf{C}_{\varepsilon / 2}} \frac{\psi_{\varepsilon}(z)-z}{z^{2}} d z\right| \leq \frac{2}{\varepsilon} \sup _{z \in \mathbf{C}_{\varepsilon / 2}}\left\{\left|\psi_{\varepsilon}(z)-z\right|\right\} \leq C_{1} \varepsilon .
$$

For the first integral, we use that $\left|\psi_{\varepsilon}(z)\right|=|z|$ if $|z| \leq \varepsilon / 2$ (see (13)), and so

$$
\left|\frac{1}{2 \pi i} \int_{\mathbf{C}_{\varepsilon / 2}} \frac{h_{\varepsilon} \circ \psi_{\varepsilon}(z)-\psi_{\varepsilon}(z)}{z^{2}} d z\right| \leq \frac{2}{\varepsilon} \sup _{z \in \mathbf{C}_{\varepsilon / 2}}\left\{\left|h_{\varepsilon}(z)-z\right|\right\} .
$$

In order to bound $h_{\varepsilon}(z)-z$, we first recall that $\psi_{\varepsilon}(z)$ is $\left(C_{1} \varepsilon\right)$-quasiconformal (see Proposition 4.9). Hence, the maximal dilatation of $\mu_{\varepsilon}$ is bounded by the same constant, that is $\left\|\mu_{\varepsilon}\right\| \leq C_{1} \varepsilon$. Now, we want to apply Proposition B. However, we cannot do it directly because $h_{\varepsilon}$ has been constructed in such a way that $h_{\varepsilon}(0)=0$ and $h_{\varepsilon}\left(c_{-}(\varepsilon)\right)=-1$, where $c_{-}(\varepsilon)=\left(-1-\sqrt{1-\varepsilon^{2}}\right) / 2$ is the critical point of $F_{\alpha(\varepsilon), \varepsilon}$ given in (10). We can arrange $h_{\varepsilon}$ to apply Proposition B simply by defining $\widetilde{h}_{\varepsilon}(z)=-h_{\varepsilon}\left(c_{-}(\varepsilon) z\right)$. We observe that $\widetilde{h}_{\varepsilon}$ is a quasiconformal map of $\mathbb{C}$, solving the Beltrami equation (7) with the Beltrami coefficient $\tilde{\mu}_{\varepsilon}(z)=\mu_{\tilde{h}_{\varepsilon}}(z)=\mu_{\varepsilon}\left(c_{-}(\varepsilon) z\right)$ and verifies $\widetilde{h}_{\varepsilon}(0)=0$ and $\widetilde{h}_{\varepsilon}(1)=1$. Then, if we assume that $\varepsilon$ is small enough such that $\left\|\tilde{\mu}_{\varepsilon}\right\|=\left\|\mu_{\varepsilon}\right\| \leq C_{1} \varepsilon \leq \rho$, we can apply Proposition B to $\tilde{\mu}_{\varepsilon}$, obtaining that if $|z| \leq \rho$ and $\left\|\tilde{\mu}_{\varepsilon}\right\||\log | z|| \leq C_{1} \varepsilon|\log | z|| \leq \rho$, then

$$
\left|\widetilde{h}_{\varepsilon}(z)-z\right| \leq C\left\|\tilde{\mu}_{\varepsilon}\right\||z||\log | z|| \leq C C_{1} \varepsilon|z||\log | z|| \text {. }
$$

Taking into account that $\left|1 / c_{-}(\varepsilon)+1\right| \leq \varepsilon^{2}$, we have that if $|z|=\varepsilon / 2$ with $\varepsilon$ small, then

$$
\left|\frac{z}{c_{-}(\varepsilon)}\right| \leq \frac{\varepsilon}{2}\left(1+\varepsilon^{2}\right) \leq \rho, \quad\left\|\mu_{\varepsilon}\right\||\log | \frac{z}{c_{-}(\varepsilon)}|| \leq C_{1} \varepsilon\left|\log \left(\frac{\varepsilon}{2}\left(1+\varepsilon^{2}\right)\right)\right| \leq \rho .
$$

So, we can apply formula $(21)$ to $z / c_{-}(\varepsilon)$, obtaining

$$
\begin{aligned}
\left|h_{\varepsilon}(z)-z\right| & =\left|\widetilde{h}_{\varepsilon}\left(\frac{z}{c_{-}(\varepsilon)}\right)+z\right| \leq\left|\widetilde{h}_{\varepsilon}\left(\frac{z}{c_{-}(\varepsilon)}\right)-\frac{z}{c_{-}(\varepsilon)}\right|+|z|\left|1+\frac{1}{c_{-}(\varepsilon)}\right| \\
& \leq C C_{1} \varepsilon\left|\frac{z}{c_{-}(\varepsilon)}\right| \log \left(\left|\frac{z}{c_{-}(\varepsilon)}\right|\right)+|z| \varepsilon^{2} \leq C^{\prime} \varepsilon^{2}|\log \varepsilon|
\end{aligned}
$$

with $C^{\prime}$ depending on $C$ and $C_{1}$. As a consequence of this, we can bound

$$
\left|\frac{1}{2 \pi i} \int_{\mathbf{C}_{\varepsilon / 2}} \frac{h_{\varepsilon} \circ \psi_{\varepsilon}(z)-\psi_{\varepsilon}(z)}{z^{2}} d z\right| \leq 2 C^{\prime} \varepsilon|\log \varepsilon|,
$$

obtaining $b(\varepsilon)=1+\mathcal{O}(\varepsilon|\log \varepsilon|)$, and therefore ending the proof of Proposition 4.8.

This concludes the proof of Theorem A, up to proving Proposition B. 


\section{Proof of Proposition B}

Our goal in this section is to prove Proposition B.

5.1. Proof of Proposition $B(a)$. For the sake of completeness, we give here a proof of this result. A different proof can be found, for instance, in [Mar].

Let us consider the following one-parameter family of Beltrami coefficients

$$
\mu_{t}=t \frac{\mu}{\|\mu\|}
$$

where $t \in \mathbb{D}$. As $\left\|\mu_{t}\right\|=|t|<1$ for all $t \in \mathbb{D}$, we can apply Theorem 2.12 and obtain a one-parameter family of integrating maps $h_{t}: \mathbb{C} \rightarrow \mathbb{C}$ fixing 0 and 1 , and such that $\partial h_{t} / \partial \bar{z}=\mu_{t}\left(\partial h_{t} / \partial z\right)$. Moreover, $h_{t}(z)$ depends analytically on $t$. Observe that, as $\mu_{t}=0$ if $t=0$ and $\mu_{t}=\mu$ if $t=\|\mu\|$, we have that $h_{0}=\operatorname{Id}$ and $h_{\|\mu\|}=h$.

Now, let $z \in \mathbb{C} \backslash\{0,1\}$ fixed, and consider the holomorphic map

$$
\begin{aligned}
f_{z}: \mathbb{D} & \longrightarrow \mathbb{C} \backslash\{0,1\} \\
t & \longmapsto h_{t}(z) .
\end{aligned}
$$

Since both $\mathbb{D}$ and $\mathbb{C} \backslash\{0,1\}$ are hyperbolic sets, we conclude from the Schwarz-Pick lemma (see Theorem 2.23) that $f_{z}$ is a contraction in the Poincare metrics, that is

$$
\delta_{\mathbb{C} \backslash\{0,1\}}\left(h_{t_{1}}(z), h_{t_{2}}(z)\right) \leq \delta_{\mathbb{D}}\left(t_{1}, t_{2}\right)
$$

for all $t_{1}, t_{2} \in \mathbb{D}$. If we take $t_{1}=0$ and $t_{2}=\|\mu\|$, the statement follows.

5.2. Proof of Proposition $B(b)$. The second part of Proposition B will be deduced from the first one by comparing the Euclidean distance between points close to 0 , with the hyperbolic distance in $\mathbb{C} \backslash\{0,1\}$, and using the explicit formula for $\delta_{\mathbb{D}}(0,\|\mu\|)$ given in Proposition 2.18. We note that it is not easy to work directly with $\delta_{\mathbb{C} \backslash\{0,1\}}$, since there is no explicit formula for this hyperbolic distance. However, as stated in the following proposition, $\delta_{\mathbb{C} \backslash\{0,1\}}$ is comparable-close to the origin-to $\delta_{\mathbb{D}^{*}}$, for which we have an explicit expression (see Proposition 2.22).

Proposition 5.1. There exist constants $0<c<1 / 2, M>0$ and $\sigma>0$ such that:

(a) for all $z_{1}, z_{2} \in \mathbb{D}_{c}^{*}=\mathbb{D}_{c} \backslash\{0\}$

$$
1 \leq \frac{\delta_{\mathbb{D}^{*}}\left(z_{1}, z_{2}\right)}{\delta_{\mathbb{C} \backslash\{0,1\}}\left(z_{1}, z_{2}\right)} \leq 1+M ;
$$

(b) if $z_{1}, z_{2} \in \mathbb{D}^{*}$, with $\left|z_{1}\right| \leq c / 2$ and $\delta_{\mathbb{C} \backslash\{0,1\}}\left(z_{1}, z_{2}\right) \leq \sigma$, then $\left|z_{2}\right| \leq c$.

The comparison between the Euclidean distance and $\delta_{\mathbb{D}^{*}}$ is given by the following lemma.

LEMMA 5.2. Let $z_{1}, z_{2} \in \mathbb{D}^{*}$ satisfying

$$
|\log | z_{1}|| \delta_{\mathbb{D}^{*}}\left(z_{1}, z_{2}\right) \leq \frac{1}{\sqrt{2}}, \quad \delta_{\mathbb{D}^{*}}\left(z_{1}, z_{2}\right) \leq 2 \log (1+\sqrt{2}),
$$

then:

$$
\left|z_{1}-z_{2}\right| \leq \frac{\sqrt{2}}{\sqrt{2}-1}\left|z_{1}\right||\log | z_{1}|| \delta_{\mathbb{D}^{*}}\left(z_{1}, z_{2}\right) .
$$


Remark 5.3. We point out that Proposition B(a) only provides information about $\delta_{\mathbb{C} \backslash\{0,1\}}(z, h(z))$ and thus on $\delta_{\mathbb{D}^{*}}(z, h(z))$ (after using Proposition 5.1). This is the reason for which the hypotheses of Lemma 5.2 are formulated in terms of the hyperbolic distance. If, for instance, a priori estimates on $\left|z_{1}-z_{2}\right| /\left|z_{1}\right|$ were known, then the statement (and the proof) of the lemma could be simplified.

The proofs of Proposition 5.1 and Lemma 5.2 are postponed to the following section. Now, we prove the second part of Proposition B.

The key point to proving (b) is the estimate provided by (a):

$$
\delta_{\mathbb{C} \backslash\{0,1\}}(z, h(z)) \leq \delta_{\mathbb{D}}(0,\|\mu\|) .
$$

Using this inequality, we check that the desired result follows by taking

$$
\rho=\min \left\{\frac{\sigma}{4}, \frac{c}{2}, \frac{\sqrt{2}}{8(1+M)}\right\}
$$

where $0<c<\frac{1}{2}, M>0$ and $\sigma>0$ are the constants provided by Proposition 5.1.

First of all, by Proposition 2.18 we have

$$
\delta_{\mathbb{D}}(0,\|\mu\|)=\log \left(\frac{1+\|\mu\|}{1-\|\mu\|}\right) .
$$

Now, as we are assuming $\|\mu\| \leq \rho$, we have $\|\mu\| \leq c / 2<\frac{1}{2}$. So, applying the mean value theorem to the logarithm, we deduce that $\delta_{\mathbb{D}}(0,\|\mu\|) \leq 4\|\mu\|$.

As we also suppose $\|\mu\| \leq \sigma / 4$, the previous inequality on $\delta_{\mathbb{D}}(0,\|\mu\|)$ jointly with (22) implies that $\delta_{\mathbb{C} \backslash\{0,1\}}(z, h(z)) \leq \sigma$. Then, from Proposition 5.1(b) we deduce that if $|z| \leq c / 2$, then $|h(z)| \leq c$. This a priori estimate on the size of $h(z)$ allows us to apply Proposition 5.1(a), obtaining

$$
\delta_{\mathbb{D}^{*}}(z, h(z)) \leq(1+M) \delta_{\mathbb{C} \backslash\{0,1\}}(z, h(z)) \leq 4(1+M)\|\mu\| .
$$

Finally, if we combine this last inequality with the hypotheses $\|\mu\| \leq \rho$ and $\|\mu\||\log | z|| \leq$ $\rho$, we obtain

$$
\delta_{\mathbb{D}^{*}}(z, h(z))|\log | z|| \leq 4(1+M)\|\mu\||\log | z|| \leq 4(1+M) \rho \leq \frac{1}{\sqrt{2}},
$$

and

$$
\delta_{\mathbb{D}^{*}}(z, h(z)) \leq 4(1+M)\|\mu\| \leq 4(1+M) \rho \leq \frac{\sqrt{2}}{2} \leq 2 \log (1+\sqrt{2}) .
$$

Thus, the hypotheses of Lemma 5.2 are verified for $z_{1}=z$ and $z_{2}=h(z)$, giving

$$
\begin{aligned}
|h(z)-z| & \leq \frac{\sqrt{2}}{\sqrt{2}-1}|z||\log | z|| \delta_{\mathbb{D}^{*}}(z, h(z)) \\
& \leq \frac{4 \sqrt{2}(1+M)}{\sqrt{2}-1}\|\mu\||z||\log | z|| \equiv C\|\mu\||z||\log | z||
\end{aligned}
$$




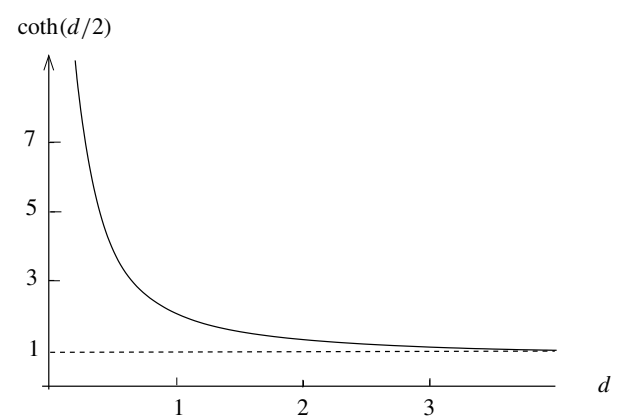

FIGURE 5 . The graph of $\operatorname{coth}(d / 2)$ or equivalently $\left(e^{d}+1\right) /\left(e^{d}-1\right)$.

5.3. Proof of Proposition 5.1 and Lemma 5.2. We recall that Ahlfors' lemma (see Proposition 2.24) provides a way to compare the line elements corresponding to the hyperbolic distances when $\mathcal{U}=\mathbb{D}^{*}$ and $\mathcal{V}=\mathbb{C} \backslash\{0,1\}$. To prove Proposition 5.1, we have to obtain a similar comparison result for the hyperbolic distances between two points. In addition to Ahlfors' lemma we need the following result, whose proof is postponed to the end of this section.

Lemma 5.4. There exists a constant $0<\kappa<1$ and a topological closed disk D, $0 \in D \subset \mathbb{D}_{1-\kappa}$, such that if $z_{1}, z_{2} \in D^{*}=D \backslash\{0\}$, then the geodesic (with respect to $\lambda \mathbb{C} \backslash\{0,1\})$ that joins $z_{1}$ and $z_{2}$ is entirely contained in $D$.

Proof of Proposition 5.1. During the proof, let us set $\mathcal{U}=\mathbb{D}^{*}$ and $\mathcal{V}=\mathbb{C} \backslash\{0,1\}$.

(a) The left-hand inequality follows from its analogue in Proposition 2.24. Indeed, let $\gamma \subset \mathcal{U}$ be a path joining $z_{1}$ and $z_{2}$. Since $\mathcal{U} \subset \mathcal{V}$, we have

$$
l_{\mathcal{U}}(\gamma)=\int_{a}^{b} \lambda_{\mathcal{U}}(\gamma(t))\left|\gamma^{\prime}(t)\right| d t \geq \int_{a}^{b} \lambda_{\mathcal{V}}(\gamma(t))\left|\gamma^{\prime}(t)\right| d t=l_{\mathcal{V}}(\gamma) \geq \delta_{\mathcal{V}}\left(z_{1}, z_{2}\right) .
$$

As this holds for any $\gamma$, we obtain $\delta_{\mathcal{U}}\left(z_{1}, z_{2}\right) \geq \delta_{\mathcal{V}}\left(z_{1}, z_{2}\right)$.

To prove the right-hand inequality we need to work a little harder. From Proposition 2.24 we only obtain, for any $\gamma \subset \mathcal{U}$ connecting $z_{1}$ and $z_{2}$,

$$
\begin{aligned}
l_{\mathcal{U}}(\gamma) & =\int_{a}^{b} \lambda_{\mathcal{U}}(\gamma(t))\left|\gamma^{\prime}(t)\right| d t \leq \int_{a}^{b} \operatorname{coth}\left(\frac{d(t)}{2}\right) \lambda \mathcal{V}(\gamma(t))\left|\gamma^{\prime}(t)\right| d t \\
& \leq \operatorname{coth}\left(\frac{d_{\gamma}}{2}\right) l_{\mathcal{V}}(\gamma)=K_{\gamma} l_{\mathcal{V}}(\gamma),
\end{aligned}
$$

where we define $d(t)=\delta_{\mathcal{V}}(\gamma(t), \partial \mathcal{U}), d_{\gamma}=\min _{t \in[a, b]} d(t)$ and $K_{\gamma}=\operatorname{coth}\left(d_{\gamma} / 2\right)$.

Note that if $\gamma$ is a curve that comes very close to $\partial \mathcal{U} \backslash \partial \mathcal{V}=\mathbf{C}_{1} \backslash\{1\}$, then $d_{\gamma}$ is a constant very close to 0 and, consequently, $K_{\gamma}$ is very close to infinity (see Figure 5). Thus, to assert that $K_{\gamma}$ is finite we need to have $\gamma$ bounded away from the set $\mathbf{C}_{1} \backslash\{1\}$.

Let $0<c<\frac{1}{2}$ be a constant such that the disc $\mathbb{D}_{c}$ is contained in the topological disk $D, D \subset \mathbb{D}_{1-\kappa}$, of Lemma 5.4. For any two points $z_{1}, z_{2} \in \mathbb{D}_{c}^{*}$ it is not difficult to check, by combining Proposition 2.19 with Proposition 2.22 , that the $\mathbb{D}^{*}$-geodesic path joining $z_{1}$ 
and $z_{2}$ is entirely contained in a disk whose radius is the maximum of the moduli of $z_{1}$ and $z_{2}$, which is at most $c$. Consequently,

$$
\delta_{\mathcal{U}}\left(z_{1}, z_{2}\right)=\inf _{\gamma \subset \mathcal{U}} l_{\mathcal{U}}(\gamma)=\inf _{\gamma \subset \mathbb{D}_{c}^{*}} l_{\mathcal{U}}(\gamma)=\inf _{\gamma \subset \mathbb{D}_{1-\kappa}^{*}} l_{\mathcal{U}}(\gamma)
$$

where the infima are always taken on paths $\gamma$ joining $z_{1}$ and $z_{2}$. Since for any $\gamma \subset \mathbb{D}_{1-\kappa}^{*}$, the constant $d_{\gamma}$ is bounded away from zero and therefore $K_{\gamma} \leq 1+M$ for a certain constant $M=M(\kappa)>0$, Equation (23) reads

$$
\inf _{\gamma \subset \mathbb{D}_{1-\kappa}^{*}} l \mathcal{U}(\gamma) \leq \inf _{\gamma \subset \mathbb{D}_{1-\kappa}^{*}} K_{\gamma} l \mathcal{V}(\gamma) \leq(1+M) \inf _{\gamma \subset \mathbb{D}_{1-\kappa}^{*}} l \mathcal{V}(\gamma)
$$

To conclude the proof we observe that for any $z_{1}, z_{2} \in \mathbb{D}_{c}^{*}$, Lemma 5.4 guarantees that the $\mathcal{V}$-geodesic joining $z_{1}$ and $z_{2}$ is entirely contained in $D \subset \mathbb{D}_{1-\kappa}$. Then, this implies that

$$
\begin{aligned}
\delta_{\mathcal{U}}\left(z_{1}, z_{2}\right) & =\inf _{\gamma \subset \mathbb{D}_{1-\kappa}^{*}} l_{\mathcal{U}}(\gamma) \leq(1+M) \inf _{\gamma \subset \mathbb{D}_{1-\kappa}^{*}} l \mathcal{V}(\gamma) \\
& =(1+M) \inf _{\gamma \subset \mathcal{V}} l \mathcal{V}(\gamma)=(1+M) \delta_{\mathcal{V}}\left(z_{1}, z_{2}\right)
\end{aligned}
$$

(b) The geometrical definition of the Poincaré metric makes this result straightforward. We just have to define

$$
\sigma=\inf _{\substack{\left|z_{1}\right|=c \\\left|z_{2}\right|=c / 2}} \delta \mathcal{V}\left(z_{1}, z_{2}\right)
$$

We stress that this proof is independent of the particular value of $0<c<\frac{1}{2}$ provided by (a).

To conclude the first part of this section, we now prove Lemma 5.4.

Proof of Lemma 5.4. The key to proving this lemma lies on the understanding of how geodesics in the thrice punctured sphere $\mathcal{V}=\mathbb{C} \backslash\{0,1\}$ look.

We first observe that the vertical line $l$ going through the point $\frac{1}{2}$ is a geodesic since it is a line of symmetry in $\mathcal{V}$. Two more geodesics can be obtained by considering the Möbius transformations of $\overline{\mathbb{C}}, g(z)=(a z+b) /(c z+d)$, that map $\mathcal{V}$ onto itself (i.e. those that permute 1,0 and $\infty)$. These maps are isometries and hence they send geodesics to geodesics. In particular, some of them map $l$ to the unit circle and the others to the circle centered at 1 with radius 1 (check this using, for instance, the transformations $(z-1) / z$ and $1 /(1-z)$ ) (see Figure 7 below).

This already states that if two points are in $\mathbb{D}^{*}$, the geodesic path that joins them must lie entirely in $\mathbb{D}^{*}$. However, this is not enough for our purposes since we need to have a domain $D$ strictly contained in $\mathbb{D}^{*}$ with the same property. Hence we need to understand more about the other geodesics. This would be easy if we knew an explicit expression for a universal covering of $\mathcal{V}$, which could be used to transfer the geodesics of the covering space, $\mathbb{D}$ or equivalently $\mathbb{H}$, to the geodesics in $\mathcal{V}$. However, such an expression does not exist, although a universal covering $\varphi: \mathbb{D} \rightarrow \mathcal{V}$ can be obtained from the so-called modular map, $\mathcal{M}: \mathbb{H} \rightarrow \mathcal{V}$. This map has been extensively studied and we refer the reader to [Be1] or $[\mathbf{C}]$ for a detailed investigation of $\mathcal{M}$. Here we only recall the main facts that lead us to specifically prove Lemma 5.4. 


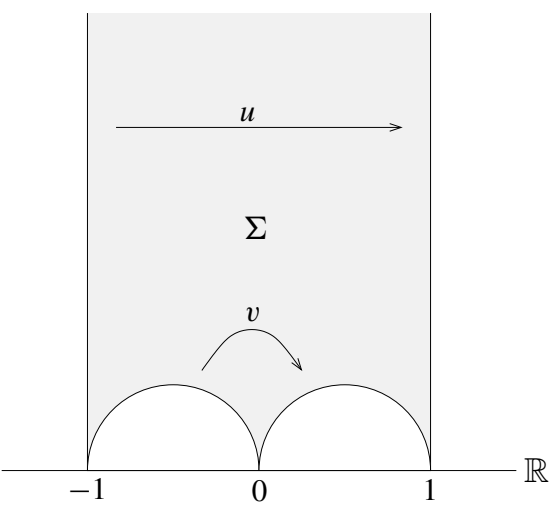

FIGURE 6. The region $\Sigma$.

Let $\Gamma$ denote the modular group, i.e. the group of Möbius transformations such that $a, d \in 2 \mathbb{Z}+1, b, c \in 2 \mathbb{Z}$ and $a d-b c=1$. Then $\Gamma$ is generated by the maps

$$
u(z)=z+2, \quad v(z)=\frac{z}{2 z+1}
$$

If we consider the region $\Sigma$ in the upper half plane depicted in Figure 6, it can be shown that $u$ and $v$ pair the sides of $\Sigma$ and that the images of $\Sigma$ by $\Gamma$ tessellate $\mathbb{H}$.

The modular map $\mathcal{M}: \mathbb{H} \rightarrow \mathcal{V}$ is constructed as follows. Consider the open righthalf piece of $\Sigma$, or more precisely $\Sigma_{0}=\Sigma \cap\{\operatorname{Re}(z)>0\}$, and choose a conformal map $\mathcal{M}: \Sigma_{0} \rightarrow \mathbb{H}$ (whose existence follows from the Riemann mapping theorem). Then, $\mathcal{M}$ extends to a homeomorphism between the boundaries of these domains and, by combining it with an appropriate conformal automorphism of $\mathbb{H}$, we may assume that $\mathcal{M}$ fixes 0,1 and $\infty$. Hence, the positive imaginary axis is mapped to the interval $(-\infty, 0)$. By the Schwarz reflection principle, we can extend $\mathcal{M}$ across this axis so that it maps the left part of $\Sigma$ to the lower half plane. So, we have that $\mathcal{M}$ (abusing notation again) conformally maps $\Sigma$ onto $\mathbb{C} \backslash[0,+\infty)$. Using the transformations $u$ and $v$ we can analytically continue $\mathcal{M}$ to the whole upper half plane, obtaining a holomorphic covering $\mathcal{M}: \mathbb{H} \rightarrow \mathcal{V}$. We can show that $\mathcal{M}(z)=\mathcal{M}(w)$ if and only if $w=g(z)$ for some $g \in \Gamma$.

Now, let us see which geodesics in $\mathbb{H}$ correspond to those we know in $\mathcal{V}$. Recall that geodesics in $\mathbb{H}$ are either vertical lines or half circles perpendicular to the real axis (see Proposition 2.19). It is not hard to check that the half circle going through $-1, i$ and 1 is mapped by $\mathcal{M}$ to the unit circle, while the vertical segments $\left\{ \pm \frac{1}{2}+i t: \frac{1}{2} \leq t \leq+\infty\right\}$ are mapped to $l$ (see Figure 7).

Note that in this setting, if we take $\gamma$ a piece of $\mathbb{H}$-geodesic joining two symmetric points on the lower boundary of $\Sigma$, it would be mapped by $\mathcal{M}$ to a simple closed curve inside $\mathbb{D}$ surrounding $0, \mathcal{M}(\gamma)$, which will be a $\mathcal{V}$-geodesic. We define $D$ as the topological disk bounded by the curve $\mathcal{M}(\gamma)$. Thus, the $\mathcal{V}$-geodesic path joining two points in $D$ lies entirely in $D$. Now, if we pick $\gamma$, for instance, the $\mathbb{H}$-geodesic connecting $\frac{1}{2}+i / 2$ and $-\frac{1}{2}+i / 2$ (see Figure 7), then the lemma is proved. 


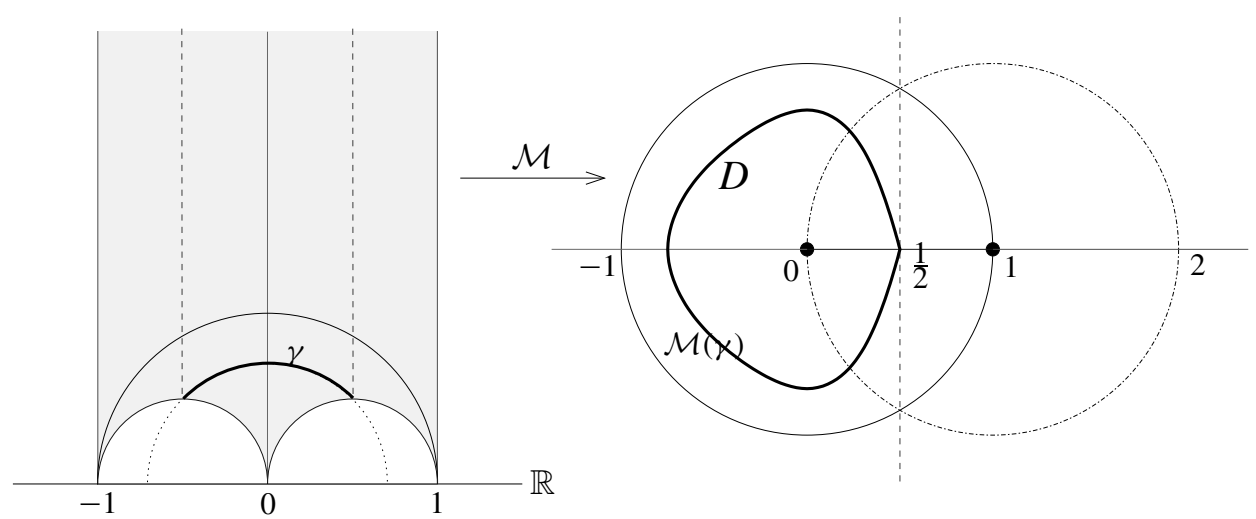

FIGURE 7. The map $\mathcal{M}: \mathbb{H} \rightarrow \mathcal{V}$, some geodesics in $\mathcal{V}$ and the topological disk $D$.

The second part of this section is devoted to proving Lemma 5.2. The proof of this lemma will need the following auxiliary results.

LEMMA 5.5. Given $z_{1}, z_{2} \in \mathbb{D}^{*}$, then we have:

(i) $\quad|\log | z_{1}|-\log | z_{2}||^{2} \leq \log \left|z_{1}\right| \log \left|z_{2}\right| \sinh ^{2}\left[\delta_{\mathbb{D} *}\left(z_{1}, z_{2}\right) / 2\right]$;

(ii) $\quad\left(\arg \left(z_{1}\right)-\arg \left(z_{2}\right)\right)^{2} \leq \log \left|z_{1}\right| \log \left|z_{2}\right| \sinh ^{2}\left[\delta_{\mathbb{D}^{*}}\left(z_{1}, z_{2}\right) / 2\right]$;

(iii) $\left|z_{1}-z_{2}\right|^{2} \leq 2\left(\max \left\{\left|z_{1}\right|,\left|z_{2}\right|\right\}\right)^{2} \log \left|z_{1}\right| \log \left|z_{2}\right| \sinh ^{2}\left[\delta_{\mathbb{D} *}\left(z_{1}, z_{2}\right) / 2\right]$.

Proof. (i) and (ii) are immediate by applying the formula for $\delta_{\mathbb{D}^{*}}\left(z_{1}, z_{2}\right)$ given in Proposition 2.22, and using that

$$
\left|\log z_{1}-\log z_{2}\right|^{2}=|\log | z_{1}|-\log | z_{2}||^{2}+\left(\arg \left(z_{1}\right)-\arg \left(z_{2}\right)\right)^{2} .
$$

On the other hand, from the cosine rule, we can write

$$
\begin{aligned}
\left|z_{1}-z_{2}\right|^{2} & =\left|z_{1}\right|^{2}+\left|z_{2}\right|^{2}-2 \cos \left(\arg \left(z_{1}\right)-\arg \left(z_{2}\right)\right)\left|z_{1}\right|\left|z_{2}\right| \\
& =\left(\left|z_{1}\right|-\left|z_{2}\right|\right)^{2}+2\left(1-\cos \left(\arg \left(z_{1}\right)-\arg \left(z_{2}\right)\right)\right)\left|z_{1}\right|\left|z_{2}\right| .
\end{aligned}
$$

Then, (iii) follows by combining this expression with (i) and (ii), the mean value theorem,

$$
|\log | z_{1}|-\log | z_{2}||=\frac{1}{\xi} \| z_{1}|-| z_{2}||, \quad \xi \in\left\langle\left|z_{1}\right|,\left|z_{2}\right|\right\rangle,
$$

and the bound

$$
1-\cos x \leq \frac{x^{2}}{2}, \quad x \in[-\pi, \pi] .
$$

The third part of Lemma 5.5 is very close to what it is stated in Lemma 5.2, but it is not exactly what we need: we want a bound for $\left|z_{1}-z_{2}\right|$ depending just on $z_{1}$ and $\delta_{\mathbb{D}^{*}}\left(z_{1}, z_{2}\right)$. This requires the following a priori estimate.

COROLlary 5.6. Given $z_{1}, z_{2} \in \mathbb{D}^{*}$ such that $\delta_{\mathbb{D}^{*}}\left(z_{1}, z_{2}\right) \leq 2 \log (1+\sqrt{2})$, we have

$$
|\log | z_{2}|| \leq \frac{3+\sqrt{5}}{2}|\log | z_{1}|| .
$$


Proof. If we set $x=-\log \left|z_{2}\right|>0$ and $d=\delta_{\mathbb{D}^{*}}\left(z_{1}, z_{2}\right)$, then from Lemma 5.5(i) we have that

$$
x^{2}+\log \left|z_{1}\right|\left(2+\sinh ^{2}\left(\frac{d}{2}\right)\right) x+\log ^{2}\left|z_{1}\right| \leq 0 .
$$

Thus, $x_{-} \leq-\log \left|z_{2}\right| \leq x_{+}$, where $x_{ \pm}$are, respectively, the two (positive) zeros for $x$ of the left-hand side of (24). This provides lower and upper bounds for $-\log \left|z_{2}\right|$, but we are only interested in the upper one:

$$
x_{+}=\frac{|\log | z_{1}||}{2}\left(2+\sinh ^{2}\left(\frac{d}{2}\right)+\sinh \left(\frac{d}{2}\right) \sqrt{\sinh ^{2}\left(\frac{d}{2}\right)+4}\right) \leq \frac{3+\sqrt{5}}{2}|\log | z_{1}||,
$$

where we have used that $d \leq 2 \log (1+\sqrt{2})$ implies $\sinh (d / 2) \leq 1$.

Proof of Lemma 5.2. In the proof we set $d=\delta_{\mathbb{D}^{*}}\left(z_{1}, z_{2}\right)$. To prove this result we have to deal with two different cases.

(i) If $\left|z_{2}\right| \geq\left|z_{1}\right|$, then from Lemma 5.5(iii) we have

$$
\left|z_{1}-z_{2}\right| \leq \sqrt{2}\left|z_{2}\right||\log | z_{1}|| \sinh \left(\frac{d}{2}\right)
$$

From here, the expression below follows:

$$
\left|z_{1}-z_{2}\right|\left(1-\sqrt{2}|\log | z_{1}|| \sinh \left(\frac{d}{2}\right)\right) \leq \sqrt{2}\left|z_{1}\right||\log | z_{1}|| \sinh \left(\frac{d}{2}\right) .
$$

We point out that from the hypotheses on the statement we have that $\cosh (d / 2) \leq \sqrt{2}$. Thus, using the mean value theorem we deduce

$$
\sqrt{2}|\log | z_{1}|| \sinh \left(\frac{d}{2}\right) \leq \sqrt{2}|\log | z_{1}|| \cosh \left(\frac{d}{2}\right) \frac{d}{2} \leq|\log | z_{1}|| d \leq \frac{1}{\sqrt{2}}
$$

From here, we obtain

$$
\left|z_{1}-z_{2}\right| \leq \frac{2}{\sqrt{2}-1}\left|z_{1}\right||\log | z_{1}|| \sinh \left(\frac{d}{2}\right) .
$$

(ii) If $\left|z_{1}\right| \geq\left|z_{2}\right|$, again applying Lemma 5.5(iii) and Corollary 5.6, we obtain

$$
\left|z_{1}-z_{2}\right| \leq \sqrt{2}\left|z_{1}\right||\log | z_{2}|| \sinh \left(\frac{d}{2}\right) \leq \frac{3+\sqrt{5}}{\sqrt{2}}\left|z_{1}\right||\log | z_{1}|| \sinh \left(\frac{d}{2}\right) .
$$

The proof ends by again applying the mean value theorem.

Acknowledgements. We wish to thank Adrien Douady and Ricardo Pérez-Marco for valuable discussions and suggestions. We also thank the anonymous referee for reading the manuscript and making helpful comments. The authors have been partially supported by the Catalan CIRIT grant 2001SGR-00070, Spanish DGES grant BFM2002-01344 (NF), Spanish MCyT/FEDER grant BFM2003-9504 (NF and TMS), Spanish MCyT/FEDER grant BFM2003-07521-C02-01 (JV), INTAS grant 2000-221 (TMS and JV), Catalan grant 2002/XT/00094 and Spanish DGICYT grant BFM2001-5237-E. 


\section{REFERENCES}

[Ah] L. Ahlfors. Lectures on Quasiconformal Mappings (Wadsworth and Brooks/Cole Mathematics Series). Wadsworth and Brooks/Cole, Advanced Books and Software, Monterey, CA, 1987.

[Ar] V. I. Arnold. Small denominators I. Mapping the circle onto itself. Izv. Akad. Nauk SSSR Ser. Mat. 25 (1961), 21-86. (Engl. transl. Amer. Math. Soc. Trans. 46(2) (1965), 213-284.)

[Ba] I. N. Baker. Wandering domains for maps of the punctured plane. Ann. Acad. Sci. Fenn. Ser. A I Math. 12 (1987), 191-198.

[Be1] A. F. Beardon. Iteration of rational functions. Complex Analytic Dynamical Systems (Graduate Texts in Mathematics, 132). Springer, New York, 1991.

[Be2] A. F. Beardon. The Geometry of Discrete Groups (Graduate Texts in Mathematics, 91). Springer, New York, 1983.

[Br1] A. D. Brjuno. Analytic form of differential equations (I). Trudy Moskov. Mat. Obšč. 25 (1971), 119-262. (Engl. transl. Trans. Moscow Math. Soc. 25 (1973), 131-288.)

[Br2] A. D. Brjuno. Analytic form of differential equations (II). Trudy Moskov. Mat. Obšc. 26 (1972), 119-239. (Engl. transl. Trans. Moscow Math. Soc. 26 (1974), 119-239.)

[BD] X. Buff and A. Douady. Le théorème d'intégrabilité des structures presque complexes. The Mandelbrot Set, Theme and Variations (London Mathematical Society Lecture Note Series, 274). Cambridge University Press, Cambridge, 2000, pp. 307-324.

[C] J. B. Conway. Functions of One Complex Variable. II (Graduate Texts in Mathematics, 159). Springer, New York, 1995.

[D] H. Davenport. Multiplicative Number Theory (Graduate Texts in Mathematics, 74), 3rd edn. Springer, New York, 2000.

[dMvS] W. de Melo and S. van Strien. One-dimensional Dynamics. Springer, Berlin, 1993.

[F] N. Fagella. Dynamics of the complex standard family. J. Math. Anal. Appl. 229(1) (1999), 1-31.

[FG] N. Fagella and L. Geyer. Surgery on Herman rings of the complex standard family. Ergod. Th. \& Dynam. Sys. 23(2) (2003), 493-508.

[G] L. Geyer. Siegel discs, Herman rings and the Arnold family. Trans. Amer. Math. Soc. 353(9) (2001), 3661-3683.

[Her1] M. Herman. Sur la conjugaison différentiable des difféomorphismes du cercle à des rotations. Inst. Hautes Études Sci. Publ. Math. 49 (1979), 5-233.

[Her2] M. Herman. Simple proofs of local conjugacy theorems for diffeomorphisms of the circle with almost every rotation number. Bol. Soc. Brasil. Mat. 16 (1985), 45-83.

[Ke] L. Keen. Dynamics of holomorphic self-maps of $\mathbf{C}^{*}$. Holomorphic Functions and Moduli, Vol. I (Berkeley, CA, 1986) (Mathematical Sciences Research Institute Publications, 10). Springer, New York, 1988, pp. 9-30.

[Ko1] J. Kotus. Iterated holomorphic maps on the punctured plane. Dynamical Systems (Sopron, 1985) (Lecture Notes in Economics and Mathematical Systems, 287). Springer, Berlin, 1987, pp. 10-28.

[Ko2] J. Kotus. The domains of normality of holomorphic self-maps of C*. Ann. Acad. Sci. Fenn. Ser. A I Math. 15(2) (1990), 329-340.

[Mak] P. M. Makienko. Iterations of analytic functions in C*. Dokl. Akad. Nauk SSSR 297(1) (1987), 3537. (Engl. transl. Sov. Math. Dokl. 36(3) (1988), 418-420.)

[MMY] S. Marmi, P. Moussa and J.-C. Yoccoz. The Brjuno functions and their regularity properties. Comm. Math. Phys. 186 (1997), 265-293.

[Mar] G. J. Martin. The distortion theorem for quasiconformal mappings, Schottky's theorem and holomorphic motions. Proc. Amer. Math. Soc. 125(4) (1997), 1095-1103.

[PM] R. Pérez-Marco. Solution complète au problème de Siegel de linéarisation d'une application holomorphe au voisinage d'un point fixe (d'aprés J.-C. Yoccoz), Séminaire Bourbaki, Vol. 1991/92. Astérisque 206 (1992), 273-310.

[Pet] C. L. Petersen. Geometry of Julia Sets (Lecture Notes, Gottingen). Gottingen, 1998.

[Pom] Ch. Pommerenke. Boundary Behaviour of Conformal Maps (Grundlehren der Mathematischen Wissenschaften, 299). Springer, Berlin, 1992.

[Ri] E. Risler. Linéarisation des perturbations holomorphes des rotations et applications. Mém. Soc. Math. Fr. 77, 1999.

[Ru1] H. Rüssmann and Kleine Nenner. II. Bemerkungen zur Newtonschen Methode. Nachr. Akad. Wiss. Göttingen Math.-Phys. Kl. II (1972), 1-20. 
[Ru2] H. Rüssmann. On the frequencies of quasi-periodic solutions of analytic nearly integrable Hamiltonian systems. Seminar on Dynamical Systems (St. Petersburg, 1991) (Progress in Nonlinear Differential Equations and Applications, 12). Birkhäuser, Basel, 1994, pp. 160-183.

[SV] T. M. Seara and J. Villanueva. Asymptotic behaviour of the domain of analyticity of invariant curves of the standard map. Nonlinearity 13 (2000), 1699-1744.

[Y1] J. C. Yoccoz. Théorème de Siegel, nombres de Bruno et polynômes quadratiques. Astérisque 231 (1995), 3-88

[Y2] J. C. Yoccoz. Analytic linearization of circle diffeomorphisms. Dynamical Systems and Small Divisors (Cetraro, 1998) (Lecture Notes in Mathematics, 1784). Springer, Berlin, 2002, pp. 125173. 